ARTICLE

Received 1 Jan 2015 | Accepted 26 Feb 2015 | Published 14 Apr 2015

\title{
Foxp1-mediated programming of limb-innervating motor neurons from mouse and human embryonic stem cells
}

\author{
Katrina L. Adams ${ }^{1,2,3}$, David L. Rousso ${ }^{1,2, \dagger}$, Joy A. Umbach ${ }^{4} \&$ Bennett G. Novitch ${ }^{1,2,3}$
}

\begin{abstract}
Spinal motor neurons (MNs) control diverse motor tasks including respiration, posture and locomotion that are disrupted by neurodegenerative diseases such as amyotrophic lateral sclerosis and spinal muscular atrophy. Methods directing $M N$ differentiation from stem cells have been developed to enable disease modelling in vitro. However, most protocols produce only a limited subset of endogenous MN subtypes. Here we demonstrate that limb-innervating lateral motor column (LMC) MNs can be efficiently generated from mouse and human embryonic stem cells through manipulation of the transcription factor Foxp1. Foxp1-programmed MNs exhibit features of medial and lateral LMC MNs including expression of specific motor pool markers and axon guidance receptors. Importantly, they preferentially project axons towards limb muscle explants in vitro and distal limb muscles in vivo upon transplantation-hallmarks of bona fide LMC MNs. These results present an effective approach for generating specific MN populations from stem cells for studying MN development and disease.
\end{abstract}

\footnotetext{
${ }^{1}$ Department of Neurobiology, David Geffen School of Medicine at UCLA, 610 Charles E Young Dr East, TLSB 3024, Los Angeles, California 90095, USA

${ }^{2}$ Eli and Edythe Broad Center of Regenerative Medicine and Stem Cell Research, University of California, Los Angeles, 610 Charles E Young Dr East, TLSB 3024, Los Angeles, California 90095, USA. ${ }^{3}$ Molecular Biology Institute Graduate Program, University of California, Los Angeles, 610 Charles E Young Dr East, TLSB 3024, Los Angeles, California 90095, USA. ${ }^{4}$ Department of Molecular and Medical Pharmacology, University of California, Los Angeles, 610 Charles E Young Dr East, TLSB 3024, Los Angeles, California 90095, USA. † Present address: Department of Molecular and Cellular Biology, Harvard University, Cambridge, Massachusetts 02138, USA. Correspondence and requests for materials should be addressed to B.G.N. (email: bnovitch@ucla.edu).
} 
T he ability to derive specialized cell types from pluripotent stem cells has allowed for novel insights into development and disease, particularly in the case of neurodegenerative diseases where specific neuronal populations are affected. To accurately model development and disease, stem cell-derived populations must fully recapitulate endogenous cell populations-both in the diversity of cell types generated and their functional behaviour. A prime example includes the differentiation of spinal motor neurons (MNs) from mouse and human embryonic stem cells (ESCs) and induced pluripotent stem cells (iPSCs), which has provided an unprecedented opportunity to model the pathogenesis of $\mathrm{MN}$ diseases such as amyotrophic lateral sclerosis (ALS) and spinal muscular atrophy ${ }^{1-5}$. However, despite these advances, in vitro MN differentiation protocols typically produce only a limited subset of endogenous $\mathrm{MN}$ populations ${ }^{6}$.

In developing mouse embryos, spinal MNs are organized into columns that innervate distinct muscle targets along the length of the body ${ }^{7-9}$. In addition to their muscle targets, motor columns are distinguished by their differential and combinatorial expression of LIM-homeodomain proteins, Hox proteins and other transcription factors ${ }^{10-13}$. The medial motor column (MMC) innervates axial muscles along the entire rostrocaudal axis. The lateral motor column (LMC) innervates limb muscles at cervical and lumbar levels. LMC MNs are further subdivided into medial $(\mathrm{LMCm})$ and lateral $(\mathrm{LMCl})$ populations that innervate the ventral and dorsal limb muscles, respectively. The hypaxial motor column (HMC) innervates respiratory muscles, including the diaphragm, intercostals and abdominal muscles at different levels of the spinal cord. Last, the preganglionic motor columns (PGC) innervate the sympathetic and parasympathetic ganglia and are present only at thoracic and sacral levels. The generation of these different MN subtypes and their subsequent assembly into motor circuits depends on the expression and function of key fate determinants in differentiating and postmitotic $\mathrm{MNs}^{7-13}$.

Most ESC to MN differentiation protocols rely on the use of retinoic acid (RA) and Sonic hedgehog (Shh) or Shh pathway agonists to mimic the natural process of $\mathrm{MN}$ formation in vivo ${ }^{14-17}$. However, these protocols appear to introduce significant bias in the formation of only select MN subtypes, predominantly MMC, with limited production of LMC MNs ${ }^{6,15}$. Spontaneous and RA-independent differentiation of ESCs have been shown to increase the diversity of MNs generated to an extent, but the efficiency of MN production tends to be reduced and few LMCl-like MNs form ${ }^{18,19}$. An alternative approach is to directly programme $\mathrm{MN}$ subtypes, taking advantage of our knowledge of the transcriptional programmes that specify different MN fates. One of the major fate determinants of LMC MNs in vivo is the Forkhead domain transcription factor Foxp1. All LMC and PGC MNs in the developing spinal cord express Foxp1, and Foxp1-mutant animals have severely reduced innervation and motor control of limb muscles ${ }^{12,13,20}$. We thus examined whether Foxp1 function could be used to direct the differentiation of limb-innervating MNs from ESCs, without compromising differentiation efficiency.

In this study, we demonstrate that Foxp1 misexpression in differentiating MNs suffices to generate bona fide LMC MNs in the mouse spinal cord and both mouse and human ESC-derived MNs in vitro. These Foxp1-programmed ESC-derived MNs express molecular markers of LMCm and $\mathrm{LMCl}$ motor columns and specific LMC motor pools, indicating conversion from an MMC to LMC fate. Foxp1-programmed MNs express LMC axon guidance receptors and preferentially project axons towards limb muscle explants in vitro, as well as distal limb muscles in chick embryos after transplantation. Last, Foxp1-programmed MNs preferentially form synapses with limb muscles in vitro and in vivo in contrast to control MMC-like MNs, which preferentially innervate axial muscles. Together, these results illustrate the feasibility of generating specific subtypes of MNs from pluripotent stem cells using a transcriptional programming approach, and the importance of MN diversity for achieving functionally distinct behaviours.

\section{Results}

Foxp1 misexpression restores LMC production in Foxp1 mutants. Previous work in our laboratory and others has shown that Foxp1 is necessary and sufficient for the generation and function of LMC and PGC MNs in the mouse spinal cord $12,13,20$. In Foxp1 mutants, LMC and PGC MNs transform into MMC and HMC MNs, illustrated by changes in molecular markers, settling position within the ventral horn of the spinal cord and axon projections ${ }^{12,13}$. Accordingly, Olig2::Cre; Foxp1 $1^{f l f l}$ mutants, in which Foxp1 is removed from $\mathrm{MN}$ progenitors, are unable to move their forelimb and hindlimb muscles due to the inability of MNs to coalesce into functional motor pools needed to form sensory-motor circuits ${ }^{20}$. In contrast, Hb9::Foxp1 transgenic mice, in which Foxp1 is misexpressed in most spinal MNs under the $\mathrm{Hb} 9$ promoter, display an increased generation of LMC and PGC MNs, and a corresponding decrease in MMC and HMC populations ${ }^{12,13}$.

To test whether the Hb9::Foxp1 transgene could direct LMC MN formation de novo, we interbred Hb9::Foxp1 mice with Foxp $1^{-/-}$mice and analysed MN formation in different mutant and transgenic allele combinations (Fig. 1). At E12.5, three distinct populations of MNs: MMC, HMC and LMC were present in the cervical spinal cord of both Foxp1 $1^{+/+}$and Foxp1 $1^{+/-}$ embryos (Fig. 1a) ${ }^{12,13}$. As previously described, Foxp $1^{-/-}$ embryos showed an almost complete loss of LMC MNs, and reciprocal expansion of $\mathrm{Hb}^{+} / \mathrm{Isl1}^{+} / \mathrm{Lhx}^{-} \mathrm{HMC} \mathrm{MNs}$ and $\mathrm{Isl1}^{+} / \mathrm{Lhx}^{+}{ }^{+}$MMC-like MNs (Fig. 1a) ${ }^{12,13}$. LMC MN formation was significantly restored in Hb9::Foxp1; Foxp1-1- embryos, with concomitant reductions in both ectopic HMC and MMC MNs (Fig. 1a,c). A similar trend was observed in the thoracic spinal cord (Fig. 1b). PGC MNs-another Foxp1-dependent population-returned in Hb9::Foxp1; Foxp1 ${ }^{-/}$embryos, coincident with a nearly complete suppression of ectopic HMC MNs (Fig. 1d). There was no significant difference in total MN number between all three genotypes, indicating that changes in $\mathrm{MN}$ subtypes were likely a result of fate conversion rather than variations in cell death and survival (Fig. 1e). Overall, these results indicate that transgenic Foxp1 expression is sufficient to generate LMC and PGC MNs and suppress ectopic MMC and HMC MN formation in a Foxp1-null background in vivo.

Foxp1-programmed ESC MNs form LMCm and LMCl subtypes. The ability of the Hb9::Foxp1 transgene to rescue Foxp1 $1^{-/-} \mathrm{MN}$ phenotypes in vivo led us to hypothesize that Foxp1 misexpression during ESC differentiation would be sufficient to generate LMC MNs in vitro. Foxp1 overexpression in neural progenitors disrupts $\mathrm{MN}$ differentiation, mimicking Foxp4 overexpression phenotypes ${ }^{21}$; highlighting the importance of overexpressing Foxp1 only in postmitotic MNs. Therefore, we derived mouse ESC lines from Hb9::Foxp1; Hb9::Green Fluorescent Protein (GFP) blastocysts. Four Hb9::Foxp1; Hb9::GFP (hereafter termed Hb9::Foxp1) ESC lines were generated, and each exhibited characteristic ESC colony appearance and pluripotency marker expression (Fig. 2a). The widely used HBG3 Hb9::GFP ESC line ${ }^{15}$, as well as newly created isogenic Hb9::GFP ESC lines, were used as controls for comparison throughout this study. All Hb9::GFP ESC lines behaved equivalently. Hb9::GFP and Hb9::Foxp1 ESC lines 
a
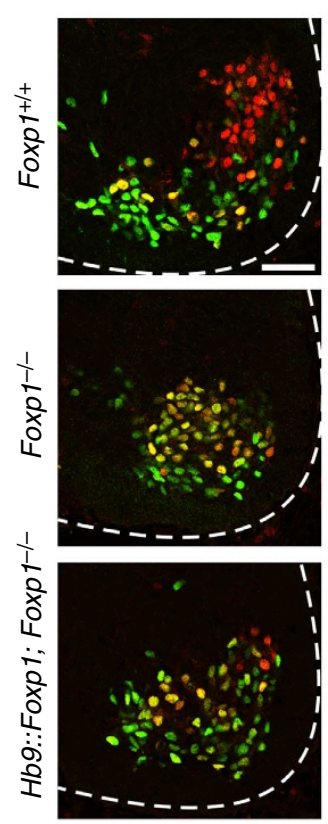

b

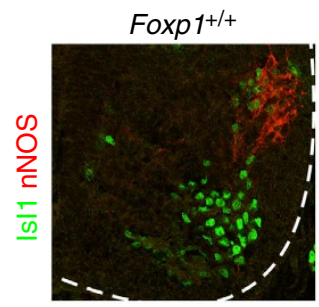

C

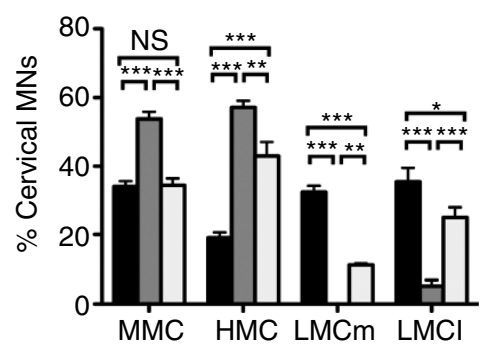

Isl1 Foxp1
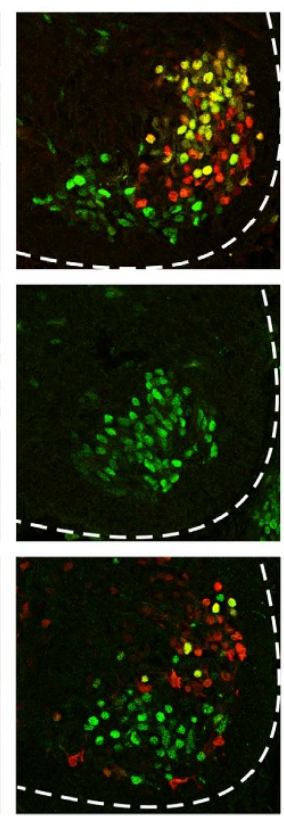

Foxp1-/-

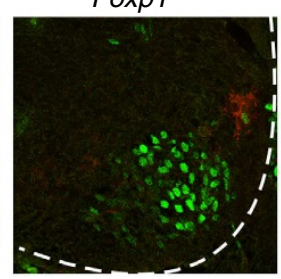

d

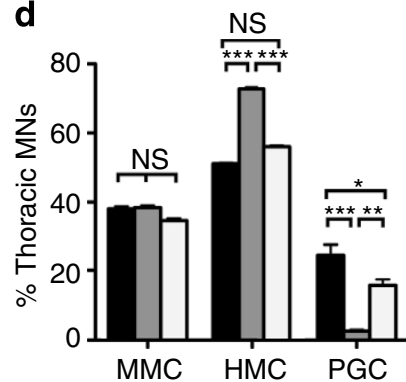

Hb9 Lhx1
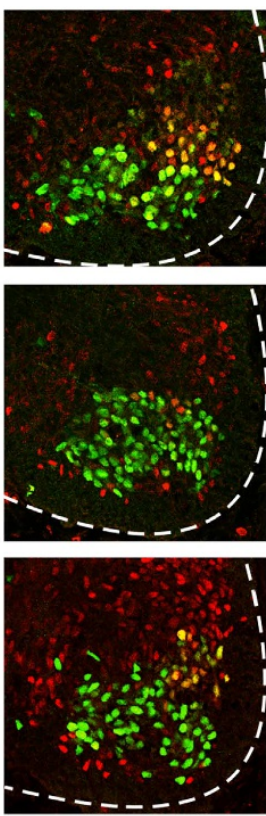

Hb9::Foxp1; Foxp1-1-

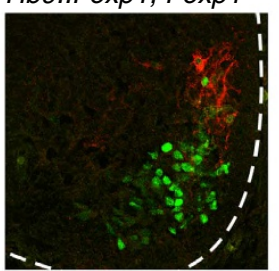

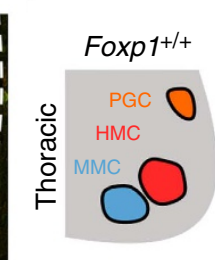

e

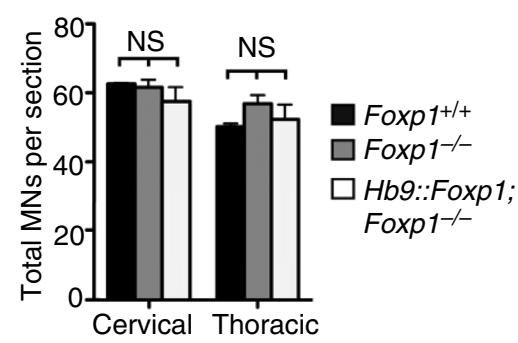

Figure 1 | Foxp1 misexpression rescues the Foxp1-mutant phenotype. Analysis of MN subtypes in the ventral horn of E12.5 Foxp ${ }^{+/}+$, Foxp ${ }^{-/-}$and Hb9::Foxp1; Foxp1 ${ }^{-/-}$littermate embryo spinal cords. Scale bar, $50 \mu \mathrm{m}$. (a) MN subtypes at cervical levels. Foxp1 ${ }^{-/-}$embryos had reduced numbers of $\mathrm{LMCm}\left(\mathrm{Is|} 1^{+} / \mathrm{Foxp}^{+}\right)$and $\mathrm{LMCl}\left(\mathrm{Hb} 9^{+} / \mathrm{Lhx}^{+}\right) \mathrm{MNs}$, while $\mathrm{HMC}\left(\mathrm{Hb} 9^{+} / \mathrm{Is|} 1^{+}\right)$and $\mathrm{MMC}$-rhomboideus $\left(|\mathrm{s}| 1^{+} / \mathrm{Lh} \times 3^{\text {low }}\right) \mathrm{MNs}$ were expanded compared with Foxp1 ${ }^{+/+}$embryos ${ }^{13}$. Hb9::Foxp1; Foxp1 ${ }^{-/-}$embryos had partially restored $\mathrm{LMCm}$ and $\mathrm{LMCl} \mathrm{MNs}$ that resided at the correct lateral positions, and reduced numbers of ectopic HMC and MMC-Rb MNs, compared with Foxp ${ }^{-/-}$embryos. Rb, rhomboideus. (b) MN subtypes at thoracic levels. The loss of PGC (IsI1+/nNOS $\left.{ }^{+}\right)$MNs in Foxp1-/- embryos was rescued in Hb9::Foxp1; Foxp1-/- embryos. (c) Quantification of MN percentages at cervical levels (mean \pm s.e.m.; $n=3$ sections averaged per embryo, 3 embryos per genotype; two-way analysis of variance (ANOVA) with Bonferroni adjustment). MMC MNs: ${ }^{\star \star \star} P<0.0001$. HMC MNs: ${ }^{\star \star \star} P<0.0001$ and ${ }^{\star \star} P=0.001$. LMCm MNs: ${ }^{\star \star \star} P<0.0001$ and ${ }^{\star \star} P=0.006$. LMCl MNs:

${ }^{\star \star \star} P<0.0001$ and ${ }^{\star} P=0.01$. NS, not significant, $P>0.5$. (d) Quantification of MN percentages at thoracic levels (mean \pm s.e.m.; $n=3$ sections averaged per embryo, 3 embryos per genotype; two-way ANOVA with Bonferroni adjustment). HMC MNs: ${ }^{\star \star \star} P<0.0001$. PGC MNs: ${ }^{\star \star \star} P<0.0001,{ }^{\star \star} P=0.0001$ and ${ }^{\star} P=0.0023$. NS, not significant, $P>0.5$. (e) Quantification of total number of MNs per section (mean \pm s.e.m.; $n=3$ sections averaged per embryo, 3 embryos per genotype; two-way ANOVA with Bonferroni adjustment). NS, not significant. $P>0.5$.

generated similar numbers of $\mathrm{GFP}^{+} / \mathrm{Hb}^{+}$MNs, indicating that Foxp1 misexpression did not affect $\mathrm{MN}$ production efficiency (Fig. 2a). On average, $40 \%$ of all ESC-derived cells expressed GFP, as determined by immunohistochemistry and fluorescenceactivated cell sorting (FACS) analysis. Hb9::Foxp1 ESC-derived MNs expressed 15- to 20-fold higher levels of Foxp1a coding messenger RNA (mRNA), compared with all Hb9::GFP control MNs. Hb9:: Foxp1 ESC-derived MNs also showed a $2.86 \pm 0.02$ fold increase (mean \pm s.e.m.) in levels of Foxp $13^{\prime}$ untranslated region (UTR) mRNA expression compared with Hb9::GFP ESCderived MNs (Fig. 2e), indicating activation of the endogenous Foxp1 locus. Foxp1 $3^{\prime}$ UTR mRNA levels were also reduced in 
a
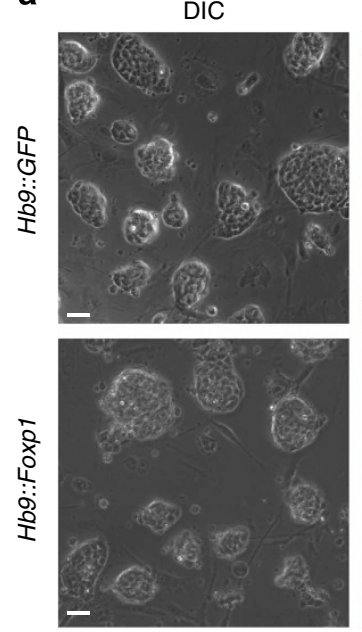

b
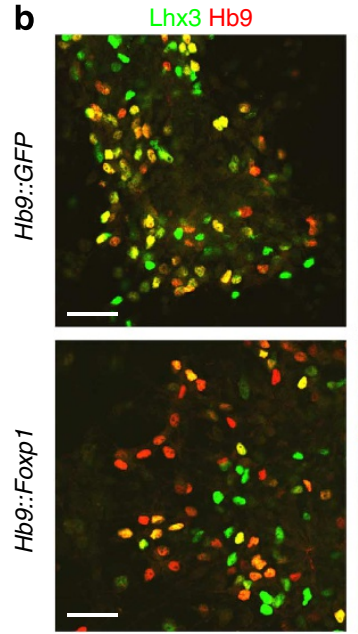

C

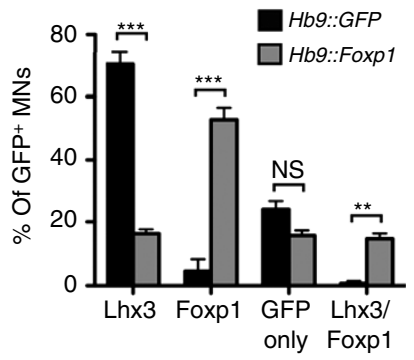

Sox2 Pou5f1 DIC
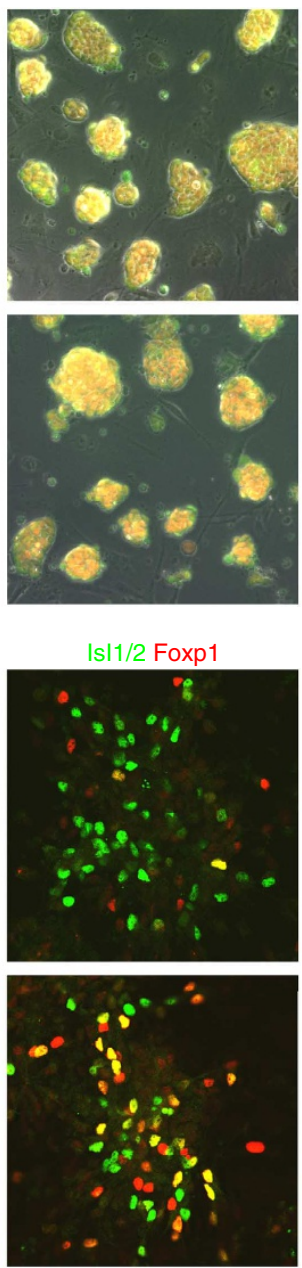

d

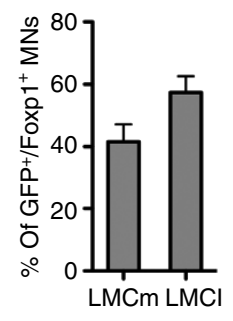

GFP Hb9
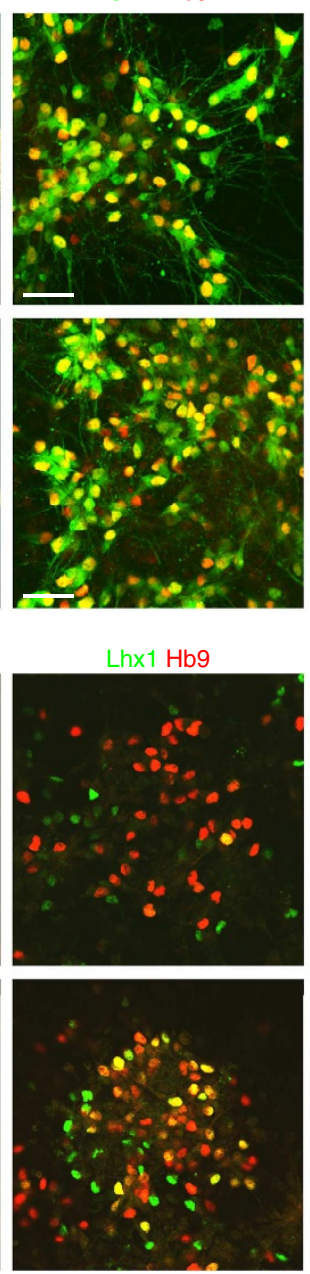

e

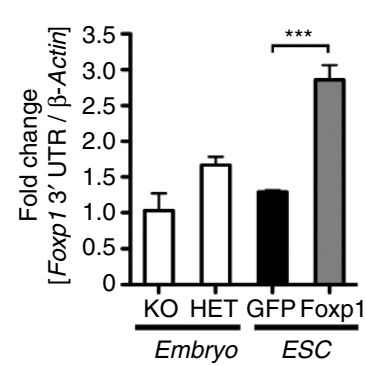

GFP Foxp1
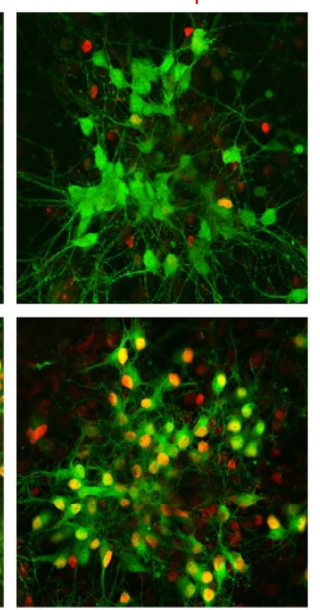

Isl1/2 Aldh1a2
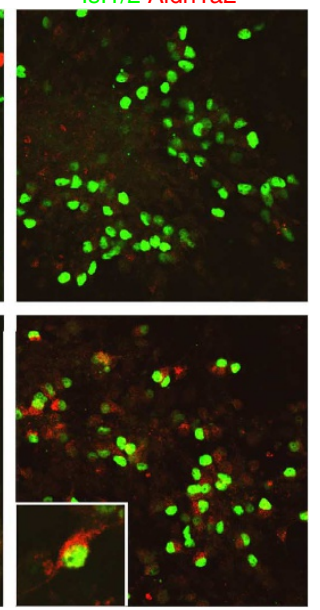

f

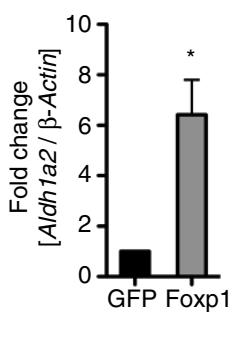

Figure 2 | Hb9:+Foxp1 ESCs generate LMCm and LMCI MNs. (a) Newly derived ESC lines displayed characteristic ESC morphology and expressed pluripotency markers. Both Hb9::GFP and Hb9:::Foxp1 ESC lines differentiated into GFP+/Hb9+ MNs, but only Hb9::Foxp1 ESC-derived MNs expressed high levels of Foxp1 protein. Scale bars, $50 \mu \mathrm{m}$. (b) Analysis of MN columnar marker expression in ESC-derived MNs. The majority of control Hb9::GFP ESC-derived MNs expressed Lhx3 and low levels of Foxp1, Lhx1 and Aldh1a2. Hb9::Foxp1 ESC-derived MNs expressed reduced levels of Lhx3 and increased levels of $\mathrm{LMCm}\left(\mathrm{Is|} 1^{+} / \mathrm{Foxp}^{+}\right)$and $\mathrm{LMCl}\left(\mathrm{Hb} 9^{+} / \mathrm{Lhx} 1^{+}\right) \mathrm{MN}$ markers. Inset shows a single Aldh1a2 ${ }^{+} \mathrm{MN}$. Scale bars, $50 \mu \mathrm{m}$. (c) Quantification of MN subtypes generated by each ESC line (mean \pm s.e.m.; $n=3$ independent experiments, 373 Hb9::GFP MNs and 403 Hb9::Foxp1 MNs total; Student's t-test). Lhx3: ${ }^{\star \star \star} P=0.0002$. Foxp1: ${ }^{\star \star \star} P=0.0008$. Lhx3/Foxp1: ${ }^{\star \star} P=0.002$. NS, not significant, $P>0.5$. (d) Quantification of the percentages of Hb9::Foxp1 ESC-derived Foxp1 ${ }^{+}$MNs that express markers of $\mathrm{LMCm}\left(\mathrm{GFP}^{+} / \mathrm{Foxp}^{+}\right.$) and $\mathrm{LMCl}\left(\mathrm{GFP}^{+} / \mathrm{Foxp}^{+} / \mathrm{Lhx}^{+}\right)$) MNs (mean \pm s.e.m.; $n=3$ independent experiments, 222 Hb9::Foxp1 MNs total). (e) Endogenous Foxp1 mRNA was expressed approximately threefold higher in Hb9::Foxp1 ESC-derived MNs, compared with Hb9::GFP ESC-derived MNs. E11.5 Foxp1 ${ }^{+/-}$and Foxp $1^{-/-}$purified MNs were used as controls (mean \pm s.e.m.; $n=3$ Foxp $1^{+/-}$embryos, 3 Foxp $1^{-/-}$embryos, 3 independent RNA collections of Hb9::GFP and Hb9::Foxp1 MNs; one-way analysis of variance with Bonferroni adjustment). ${ }^{\star \star \star} P=0.001$. (f) Aldh1a2 mRNA levels were elevated approximately sixfold higher in Hb9::Foxp1 ESC-derived MNs, compared with Hb9::GFP controls (mean \pm s.e.m.; $n=5$ independent RNA collections of Hb9::GFP and Hb9::Foxp1 MNs; paired two-tailed $t$-test). ${ }^{\star} P=0.017$.

Foxp1 $1^{-/-}$embryonic MNs compared with Foxp1 ${ }^{+/-}$embryos (Fig. 2e). These data together suggest that Foxp1 promotes its own expression through a positive feedback loop.
We next analysed the ESC-derived MNs for specific motor column markers to determine whether Foxp1 misexpression alters $\mathrm{MN}$ subtype formation in vitro. In agreement with 
previously published results ${ }^{6,15}$, the majority $(70.5 \pm 3.8 \%$ mean \pm s.e.m.) of control Hb9::GFP ESC-derived MNs expressed Lhx3, a marker of MMC MNs (Fig. 2b). Only $4.6 \pm 3.7 \%$ of Hb9::GFP MNs expressed LMC markers (Foxp1, Lhx1 and Aldh1a2) 7 days after addition of RA and Smoothened agonist (SAG) (Fig. 2b,c). In contrast, Hb9::Foxp1 ESC-derived MNs displayed significantly reduced numbers of $\mathrm{Lhx} 3^{+}$cells $(16.5 \pm$ $1.4 \%$ ) and increased numbers of cells positive for $\mathrm{LMCm}$ (Isl1/Foxp1) and LMCl (Hb9/Lhx1) markers (52.78 $\pm 3.8 \%$ were Foxp $1^{+}$, and of these $57.4 \pm 5.2 \%$ expressed Lhx1 while $41.6 \pm 5.6 \%$ expressed Isl1) (Fig. 2b,c). Interestingly, $14.8 \pm 1.8 \%$

a
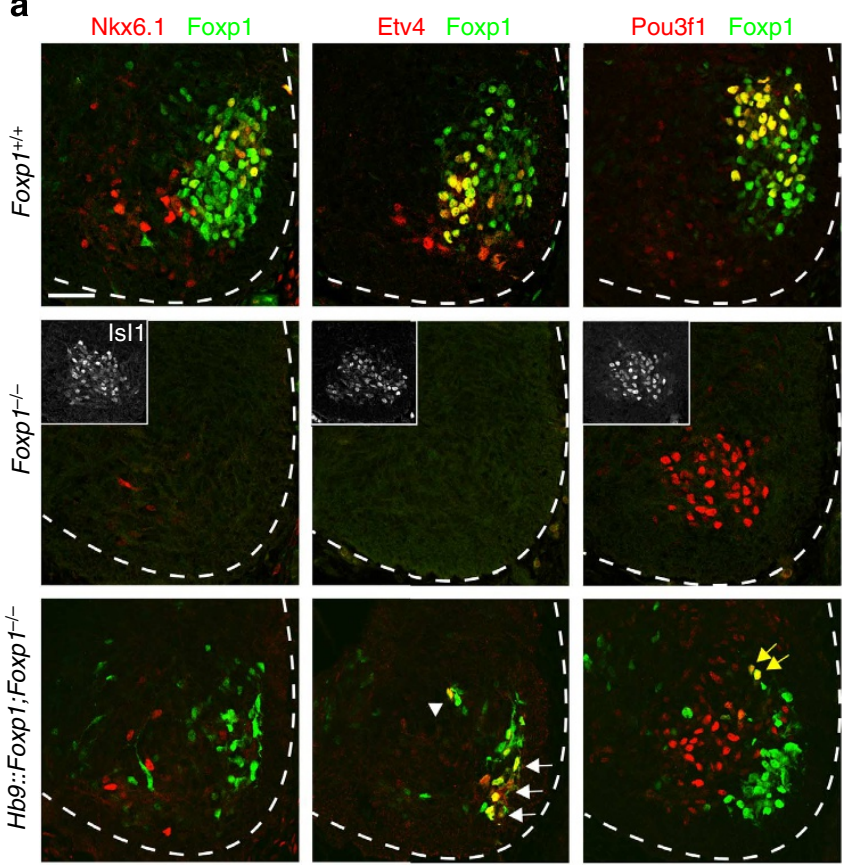

b
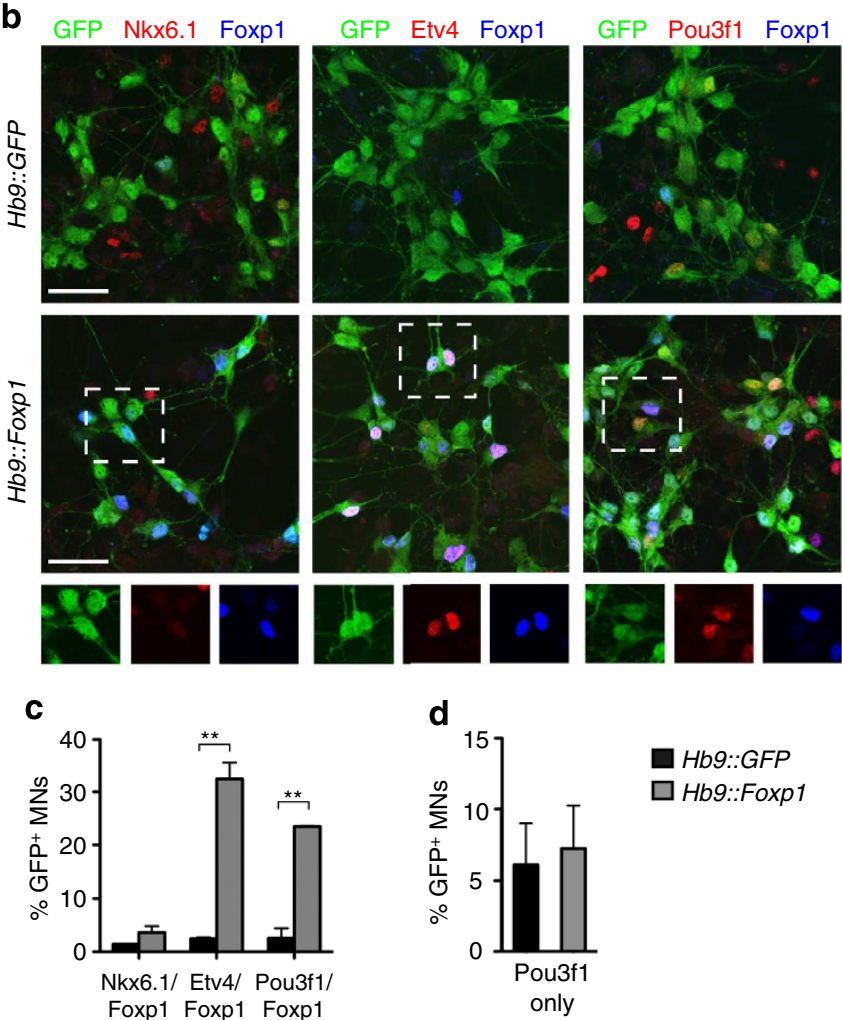

of Hb9::Foxp1 ESC-derived MNs expressed both Lhx3 and Foxp1, a cell population that is rarely found in vivo. However, this population disappeared with additional time in culture (Supplementary Fig. 1a,b), likely due to resolution in cell fates associated with the cross-repressive activities of Lhx3 and Foxp1 (refs 12,13). We did not observe any changes in the numbers of apoptotic cells assessed by activated Caspase 3 antibody staining or total MN numbers over the time in culture (Supplementary Fig. 1c,d).

We also examined ESC-derived $\mathrm{MNs}$ for expression of Aldh1a2, an RA-synthesizing enzyme expressed by LMC MNs in vivo ${ }^{22}$. Aldh1a2 mRNA levels were $6.4 \pm 1.4$-fold higher in Hb9::Foxp1 ESC-derived MNs compared with Hb9::GFP controls (Fig. 2b,f). Within the LMC-like MNs produced by the Hb9::Foxp1 ESCs, $57.4 \pm 5.2 \%$ (mean \pm s.e.m.) expressed Lhx1, suggesting a slight bias in $\mathrm{LMCl}$ formation over LMCm (Fig. 2d). This could reflect the presence of RA in the differentiation media, seeing as $\mathrm{LMCl}$ formation in vivo is influenced by retinoid signalling ${ }^{22}$. Consistent with this possibility, altering RA levels in the differentiation protocol led to corresponding changes in Lhx ${ }^{+}$Hb9::Foxp1 MNs (Supplementary Fig. 2). Together, these results indicate that Foxp1 misexpression is sufficient to transform the molecular identity of ESC-derived MNs from an MMC phenotype to an LMC phenotype.

Foxp1-programmed MNs express LMC motor pool markers. In the embryo, LMC MNs are further divided into individual motor pools, MN groups that innervate the same muscle. At cervical regions, these pools include Etv4 ${ }^{+}$(also known as Pea3) $\mathrm{LMCl}$ and $\mathrm{LMCm}$ pools that innervate the latissimus dorsi and cutaneous maximus muscles ${ }^{23,24} ; \mathrm{Nkx6.1^{+ }} \mathrm{LMCm}$ pools that innervate the biceps and other forearm flexor muscles; and a Pou3f1 ${ }^{+}$(also known as SCIP) LMCm pool that innervates the flexor carpi ulnaris muscle of the lower forearm (Fig. 3a) ${ }^{24,25}$. In vivo, the expression of these pool markers is initiated by exposure of $\mathrm{MN}$ growth cones to signals emanating from the surrounding peripheral tissue and developing muscles. For example, Etv4 is expressed in LMC MNs on exposure to glia-derived neurotrophic factor (GDNF) as LMC MN axons approach the developing limbs ${ }^{26}$. In Foxp $1^{-1-}$ embryos, there is a complete loss of Etv $4^{+}$ and $\mathrm{Nkx} 6.1^{+}$LMC motor pools at cervical levels, and a conversion of Pou3f1 ${ }^{+}$LMC MNs to Pou3f1 ${ }^{+}$HMC MNs $(\text { Fig. 3a })^{12,13}$. To test whether Foxp1 misexpression in MNs could

Figure 3 | Foxp1 misexpression induces LMC motor pool markers. (a) Analysis of cervical LMC motor pools in E12.5 Foxp1 ${ }^{+/+}$, Foxp1 ${ }^{-/-}$ and Hb9::Foxp1; Foxp1-/- embryos. All LMC motor pools were lost in Foxp $1^{-/-}$embryos. The Pou3f1 ${ }^{+}$MNs in Foxp $1^{-/-}$embryos represent the expanded HMC MN population (identified by their medial position). Insets depict Islet1 co-staining of the same section. The Etv4 motor pool was restored in Hb9::Foxp1; Foxp1-/- embryos (white arrows), with some Foxp $1^{+} /$Etv4 ${ }^{+}$MNs located more dorsally (arrowhead). The Nkx6.1 and Pou3f1 motor pools were not restored in Hb9::Foxp1; Foxp1-/- embryos, although a small number of Foxp ${ }^{+} /{\text {Pou } 3 f_{1}+}^{+}$MNs were identified (yellow arrows). Scale bar, $50 \mu \mathrm{m}$. (b) Control Hb9::GFP ESC-derived MNs did not express any LMC motor pool markers. Hb9::Foxp1 ESC-derived MNs expressed Etv4 and Pou3f1 at high levels, but did not express Nkx6.1. Boxed regions are depicted as single-colour images below each image. Scale bars, $50 \mu \mathrm{m}$. (c) Quantification of motor pool markers in ESC-derived MNs (mean \pm s.e.m.; $n=2$ independent experiments, 938 Hb9::GFP MNs and 723 Hb9::Foxp1 MNs total; Student's $t$-test). Etv4: ${ }^{\star \star} P=0.009$. Pou3f1: ${ }^{\star \star} P=0.008$. (d) Foxp1 misexpression did not alter the percentage of Pou3f1 ${ }^{+} /$Foxp $^{-}$ESC-derived HMC-like MNs (mean \pm s.e.m.; $n=3$ independent experiments, 390 Hb9::GFP MNs and 255 Hb9::Foxp1 MNs total). 
rescue these lost LMC motor pools, we analysed Hb9::Foxp1; Foxp $1^{-1-}$ embryos for expression of pool markers. Interestingly, Etv ${ }^{+}$motor pools were significantly restored, while $\mathrm{Nkx6.1^{+ }}$ and Pou3 $\mathrm{fl}^{+}$LMC motor pools were not (Fig. 3a). This deficiency may be attributed to the fact that both $\mathrm{Nkx} 6.1^{+}$and Pou $3 \mathrm{f1}{ }^{+}$LMC motor pools are associated with the LMCm, which was only partially restored in Hb9::Foxp1; Foxp1 $1^{-1-}$ embryos (Fig. 1a,c).

To determine whether Foxp1 misexpression results in LMC motor pool formation in vitro, we analysed Hb9::GFP and Hb9::Foxp1 ESC-derived MNs for these same pool markers. Control Hb9::GFP ESC-derived MNs did not express Etv4, Nkx6.1 or Pou3f1 on day 7 of differentiation, despite the presence of GDNF in the culture medium (Fig. 3b). Conversely, Hb9::Foxp1 ESC-derived MNs expressed both Etv4 and Pou3f1 (mean \pm s.e.m. $=32.4 \pm 3.1 \%$ and $23.5 \pm 0.14 \%$ of $\mathrm{GFP}^{+} \mathrm{MNs}$, respectively), but did not express $\mathrm{Nkx6.1}$ despite the clear presence of $\mathrm{LMCm} \mathrm{MNs}$ in the culture (Fig. 3b). The factor(s) required to generate $\mathrm{Nkx} 6.1^{+} \mathrm{LMC}$ motor pools are not known, and we surmise that such factors are likely missing from the culture media. To determine whether the addition of neurotrophic factors to the MN cultures biases the types of motor pools generated, we analysed Hb9::Foxp1 ESC-derived MNs cultured with and without GDNF. GDNF removal reduced the numbers of Etv4 ${ }^{+}$MNs formed, but did not affect expression of other pool markers (Supplementary Fig. 3a,b). We also confirmed that Pou3f1 ${ }^{+} / \mathrm{Foxp}^{+}$MNs did not express Lhx1, indicating an LMCm phenotype. Etv $4^{+} /$Foxp $1^{+}$MNs were more complex, with some cells expressing and others lacking Lhxl, suggesting the formation of both $\mathrm{LMCl}$ and LMCm pools (Supplementary Fig. 3c). A small number of Pou3f1 ${ }^{+} /$Foxp $^{-}$GFP $^{+}$MNs were also identified in both $H b 9:: G F P$ and $H b 9::$ Foxp1 ESC-derived MN cultures, which likely represent HMC MNs (Fig. 3d) ${ }^{13,27,28}$.

We next examined whether Foxp1 misexpression had an impact on Hox proteins, which are implicated in both columnar and pool identities ${ }^{25,29}$, but did not observe any significant differences in the cultures. The majority of both $H b 9:: G F P$ and Hb9::Foxp1 ESC-derived MNs expressed Hoxa5 and Hoxc6, which are characteristic of rostral forelimb MNs, and did not express Hoxc8 or Hoxa9 associated with more caudal MN subtypes (Supplementary Fig. 4). These data indicate that the ability of Foxp1 misexpression to convey LMC motor pool identities is most likely independent of changes in Hox activities.

Last, we tested whether Foxp1 misexpression could similarly enhance LMC MN production from human ESCs. H9 ESCs were first differentiated into MNs and then transduced with lentiviruses encoding red fluorescent protein (RFP) alone or together with the human FOXP1 gene. MNs infected with the RFP-only virus predominantly expressed the MMC marker LHX3 along with general $\mathrm{MN}$ differentiation markers such as ISL1 and choline acetyltransferase (CHAT) (Fig. 4a,c). A small population of endogenous $\mathrm{LMC}$-like FOXP1 ${ }^{+} \mathrm{CHAT}^{+} \mathrm{MNs}$ was detectable under these control cultures, though $\mathrm{LHX}^{+}{ }^{+} \mathrm{MNs}$ were scarce (Fig. 4a,c). However, when human ESC-derived MNs were infected with the virus containing FOXP1, LHX3 expression declined while LHX1 levels became readily detectable (Fig. 4a,c). These FOXP1transduced MNs further expressed LMC motor pool markers including ETV4, POU3F1 and NKX6.1 (Fig. 4b,d), recapitulating the results seen with the misexpression of Foxp1 in mouse ESCderived MNs. Thus, Foxp1 expression appears to be able to impart an LMC identity to ESC-derived MNs in human cells as well.

Foxp1-programmed MNs preferentially project to limb muscles. It has been previously shown that ESC-derived MNs do not stably innervate developing limb muscles likely due to their lack of LMC
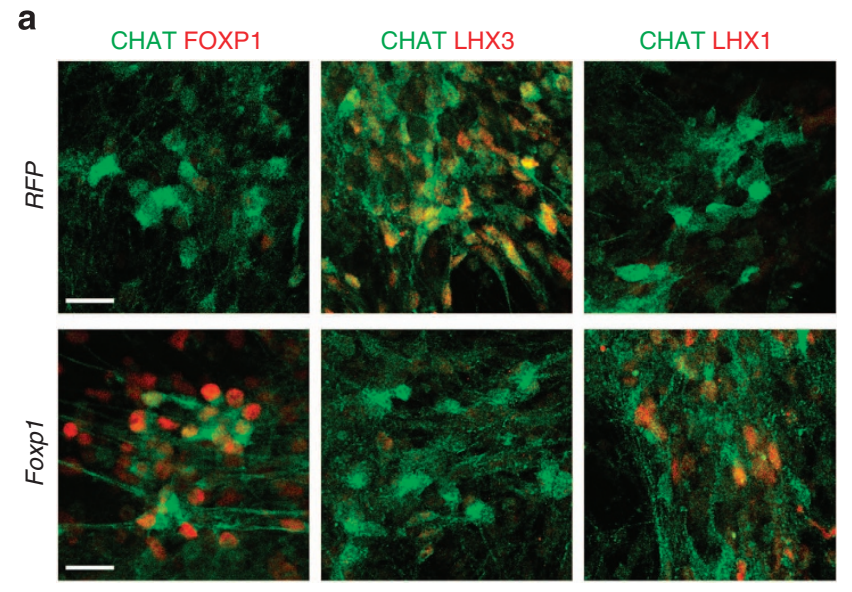

b
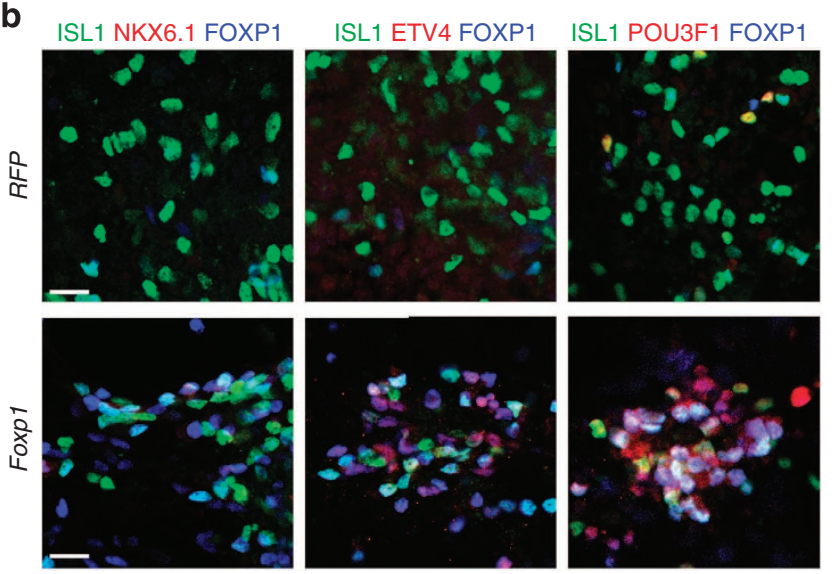

C
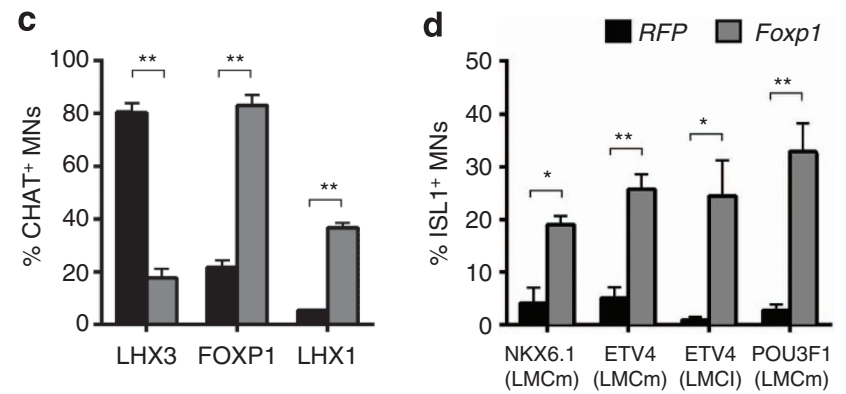

Figure 4 | FOXP1 promotes LMC MN formation from human ESCs.

(a) Control RFP-transduced human ESC-derived MNs (identified by CHAT expression) expressed high levels of LHX3 and low levels of FOXP1 and LHX1. FOXP1-transduced human ESC-derived MNs expressed increased levels of LHX1 and decreased levels of LHX3. (b) Control RFP-transduced human ESC-derived MNs did not express high levels of known LMC motor pool markers such as ETV4, POU3F1 and NKX6.1. By contrast, many FOXP1transduced human ESC-derived MNs expressed ETV4 or POU3F1, with a smaller number expressing NKX6.1. (c) Quantification of MN subtype markers in transduced human ESC-derived MNs (mean \pm s.e.m.; $n=2$ independent experiments, 700 RFP-transduced MNs and 655 FOXP1transduced MNs total; Student's $t$-test). LHX3: ${ }^{\star \star} P=0.0055$. FOXP1: ${ }^{\star \star} P=0.0056$. LHX1: ${ }^{\star \star} P=0.0035$. (d) Quantification of LMC motor pool markers in transduced human ESC-derived MNs (mean \pm s.e.m.; $n=3$ independent experiments, 2342 RFP-transduced MNs and 1624 FOXP1transduced MNs total; Student's t-test). NKX6.1: ${ }^{\star} P=0.0108$. ETV4 (LMCm): ${ }^{\star \star} P=0.0042$. ETV4 (LMCl): ${ }^{\star} P=0.0263$. POU3F1: ${ }^{\star \star} P=0.0053$ NKX6.1, ETV4 (LMCm) and POU3F1 motor pools were identified by colocalization of ISL1, FOXP1 and the respective motor pool marker. The ETV4 ( $\mathrm{LMCl}$ ) motor pool was identified by co-localization of FOXP1 and ETV4. 

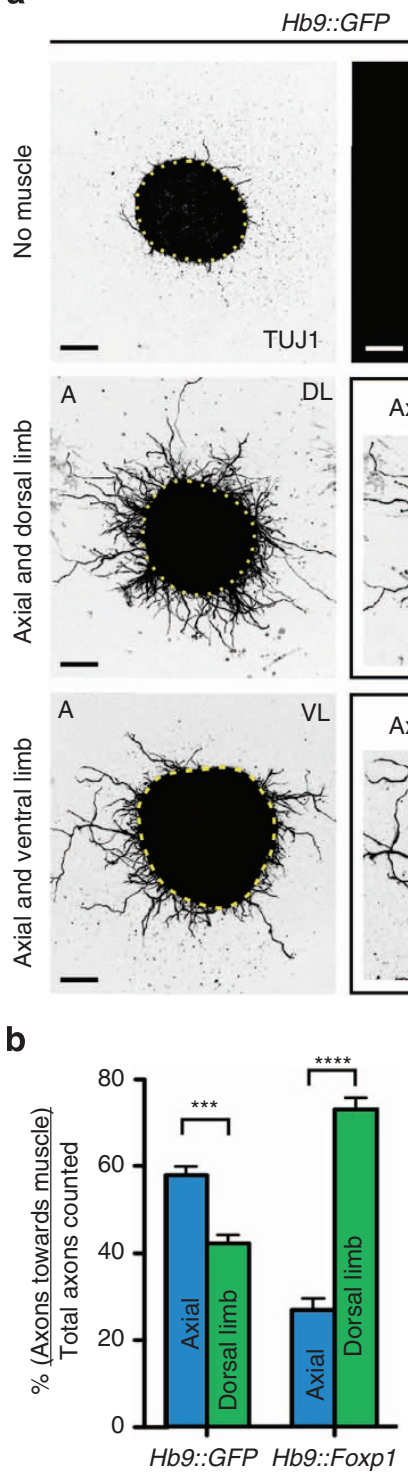

e

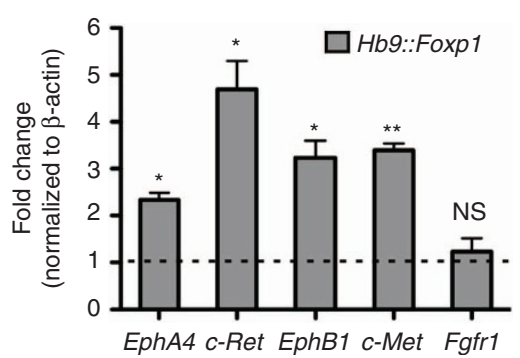

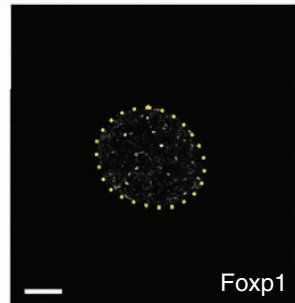
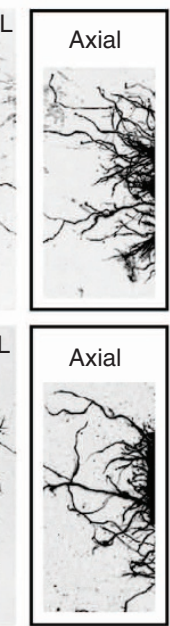

C

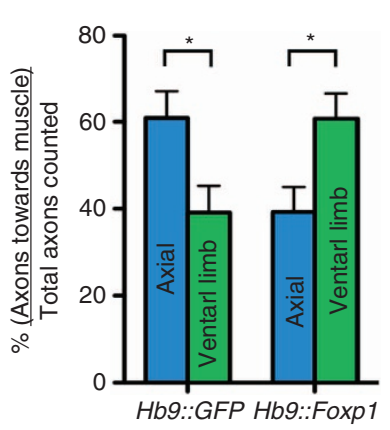

Hb9::Foxp1
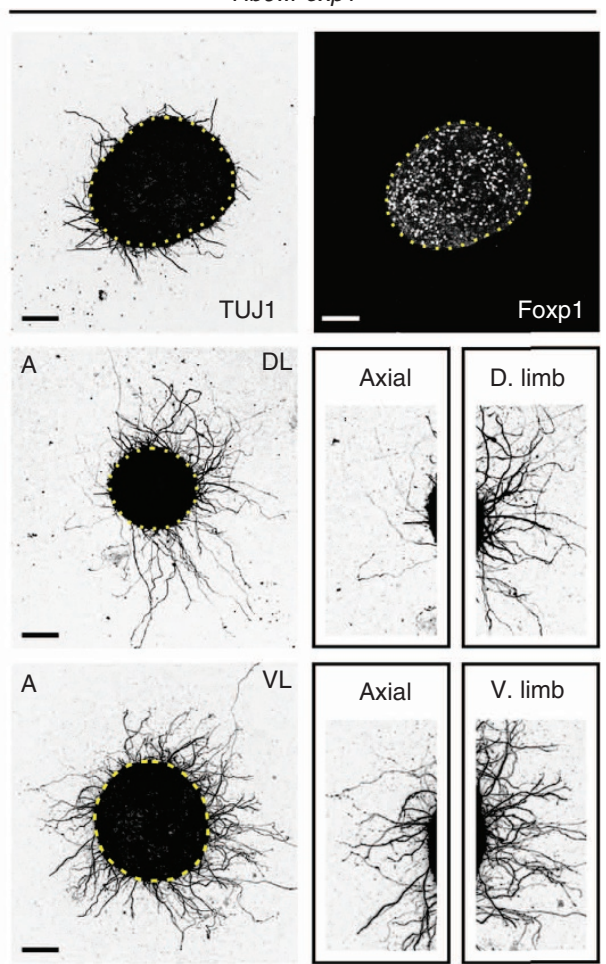

VL
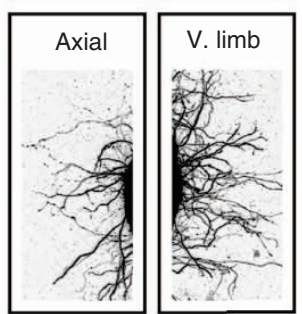

d

f
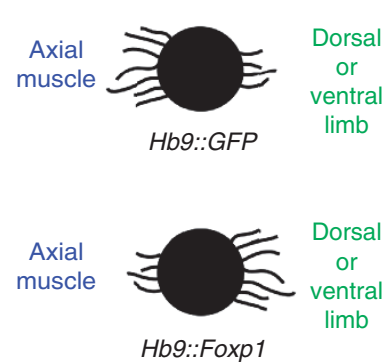

$H b 9:: G F P$
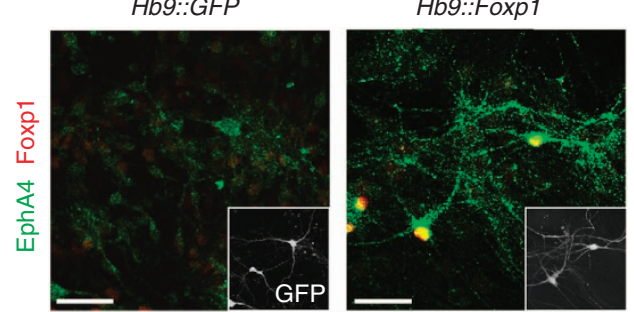

Figure 5 | Hb9::Foxp1 MNs project axons towards limb muscle explants in vitro. (a) Three-dimensional collagen cultures of EBs and different chick muscle explants. Hb9::GFP EBs projected more axons towards axial muscle than dorsal or ventral limb muscle. Hb9::Foxp1 EBs projected more axons towards dorsal and ventral limb muscle than axial muscle explants. Scale bars, $100 \mu \mathrm{m}$. 'A' $=$ axial muscle. 'DL' and 'D. limb' $=$ dorsal limb muscle. 'VL' and 'V. limb' = ventral limb muscle. (b) Quantification of Hb9::GFP and Hb9::Foxp1 axon projections when cultured between axial and dorsal limb explants (mean \pm s.e.m.; $n=5$ cultures per cell line; Student's $t$-test). ${ }^{\star \star \star} P=0.0005$. ${ }^{\star \star \star \star} P<0.0001$. (c) Quantification of Hb9::GFP and Hb9::Foxp1 axon projections when cultured between axial and ventral limb explants (mean \pm s.e.m.; $n=5$ cultures per cell line; Student's $t$-test). ${ }^{*} P=0.0465$ for $H b 9:: G F P$ and ${ }^{\star} P=0.0386$ for Hb9:::Foxp1. (d) Schematic summary of muscle explant results. (e) Hb9::Foxp1 ESC-derived MNs expressed increased levels of EphA4, c-Ret, EphB1 and c-Met mRNA, compared with Hb9::GFP ESC-derived MNs. There was no change in Fgfr1 expression (mean \pm s.e.m.; $n=3$ independent RNA collections of Hb9::GFP and Hb9::Foxp1 MNs; paired two-tailed $t$-test). ${ }^{\star} P=0.0115$ for EphA4, ${ }^{\star} P=0.0264$ for EphB1, ${ }^{\star} P=0.0258$ for $c$-Ret, ${ }^{\star \star} P=0.0037$ for $c$-Met. NS, not significant, $P>0.5$. (f) Hb9::Foxp1 ESC-derived MNs expressed higher levels of EphA4 compared with control Hb9::GFP ESC-derived MNs. Insets depict GFP staining of ESC-derived MNs. Scale bars, $50 \mu \mathrm{m}$. 
character ${ }^{6}$. To determine whether Foxp1 misexpression in ESCderived $\mathrm{MNs}$ alters axon projections, we embedded $\mathrm{GFP}^{+}$ embryoid bodies (EBs) in three-dimensional collagen beds between different chick embryo muscle explants and analysed axon outgrowth (Supplementary Fig. 5a). When Hb9::GFP and Hb9::Foxp1 EBs were cultured alone, very few axons projected into the surrounding collagen and most were very short in length (Fig. 5a). However, when Hb9::GFP EBs were cultured between axial and dorsal hindlimb muscle explants, the majority of axons projected towards the axial muscle (mean \pm s.e.m. $=56.4 \pm 1.8 \%$ axial versus $43.6 \pm 1.8 \%$ dorsal limb) (Fig. 5b,c). This trend was also seen when Hb9::GFP EBs were cultured between axial and ventral hindlimb muscle explants (mean \pm s.e.m. $=60.9 \pm 6.2 \%$ axial versus $39.1 \pm 6.2 \%$ ventral limb) (Fig. 5b,c). In contrast, a significant percentage of $H b 9::$ Foxp $1 \mathrm{~EB}$ axons projected towards dorsal hindlimb muscle (mean \pm s.e.m. $=26.8 \pm 2.7 \%$ axial versus $73.2 \pm 2.7 \%$ dorsal limb) or ventral hindlimb muscle (mean \pm s.e.m. $=39.3 \pm 5.8 \%$ axial versus $60.7 \pm 5.8 \%$ ventral limb) when cultured together with axial muscle explants (Fig. 5a-d). Similar results were observed when Hb9::Foxp1 EBs were cultured between axial and forelimb muscle explants (mean \pm s.e.m. $=36.1 \pm 3.4 \%$ axial versus $63.9 \pm 3.4 \%$ forelimb), indicating that Hb9::Foxp1 motor axons were comparably attracted to both forelimb and hindlimb muscles (Supplementary Fig. 5b,c).

These differences in axon projections suggest changes in $\mathrm{MN}$ detection of guidance and/or outgrowth cues present in the muscle explants. We therefore analysed control Hb9::GFP and Hb9::Foxp1 ESC-derived MNs for expression of known LMC axon guidance receptors. Hb9::Foxp1 MNs expressed increased levels of the EphA4 and c-Ret receptors, which are highly expressed by $\mathrm{LMCl} \mathrm{MNs}$ and critical for dorsal limb innervation in vivo (Fig. 5e,f) ${ }^{30-32}$. Hb9::Foxp1 MNs also expressed increased levels of EphB1, which is expressed by embryonic LMCm MNs and important for ventral limb innervation (Fig. 5e) ${ }^{33}$. In addition, Hb9::Foxp1 MNs expressed increased levels of $c$-Met, which is critical for both dorsal and ventral limb innervation (Fig. 5e $)^{34,35}$. However, there was no significant difference in Fgfrl levels, which are important for MMC projections towards developing axial muscles ${ }^{36}$, possibly due to the residual population of $\mathrm{Lhx} 3^{+}$MNs present in Hb9::Foxp1 MN cultures (Fig. 5e). Together, these results provide evidence that Foxp1 misexpression in ESC-derived MNs alters axon projections in vitro to favour innervation of limb muscles over axial muscles, most likely due to increased expression of LMC axon guidance receptors. It is important to note that while increased levels of EphA4 and EphB1 are consistent with a switch to an LMC identity, these molecules are not likely to be responsible for the axon projections towards limb muscle explants in vitro. These receptors and ligands are membrane bound ${ }^{37}$ and would therefore not diffuse through the collagen bed. We surmise that diffusible guidance cues, such as HGF and GDNF, likely direct Hb9::Foxp1 MNs towards limb muscles in the in vitro setting.

Foxp1-programmed MNs form synapses with limb muscles in vitro. To determine whether these changes in muscle preference were also observed at the neuromuscular junction level, we generated ESC-derived MN and muscle co-cultures. Axial and dorsal limb muscle from chick embryos were dissociated and cultured as single myotubes for several days prior to the addition of ESC-derived MNs. Cultures were analysed for synapse formation using Alexa ${ }^{647}$-conjugated alpha-bungarotoxin ( $\left.\alpha \mathrm{BTX}\right)$ to label neuromuscular junctions (Fig. 6a). $\mathrm{GFP}^{+} / \alpha \mathrm{BTX}^{+}$ synapses were identified in all cultures, regardless of muscle type. To analyse the frequency of synapse formation with different muscle types, we calculated the percentage of myotubes with at least one $\mathrm{GFP}^{+} / \alpha \mathrm{BTX}^{+}$synapse, over the total number of myotubes contacted by $\mathrm{GFP}^{+}$axons. Control Hb9::GFP MNs plated on axial myotubes resulted in a higher percentage (mean \pm s.e.m. $=$ $53.7 \pm 0.3 \%)$ of myotubes with $\mathrm{GFP}^{+} / \alpha \mathrm{BTX}^{+}$synapses, compared with limb myotube co-cultures (mean \pm s.e.m. $=$ $40.4 \pm 2.3 \%$ ) (Fig. 6b). The opposite trend was seen with Hb9::Foxp1 MNs (mean \pm s.e.m. $=41.7 \pm 3.6 \%$ axial versus $58.9 \pm 2.8 \%$ limb) (Fig. 6a,b). Furthermore, we observed a significant increase in size of the neuromuscular junctions when Hb9::Foxp1 MNs were cultured with limb muscle, compared with Hb9::GFP MNs (Fig. 6c). However, there was no difference in synapse area between the two cell lines when they were cultured with axial muscle.

Last, we performed whole-cell patch clamp recordings of the Hb9::GFP and Hb9::Foxp1 ESC-derived MNs to assess whether they displayed any differences in functional maturation (Fig. 6d,e). Both Hb9::GFP and Hb9::Foxp1 MNs robustly fired multiple action potentials (APs) on depolarizing current injection as expected for mature MNs, without any disparities in AP

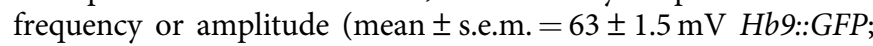
$60 \pm 2.5 \mathrm{mV}$ Hb9::Foxp1). These results indicate that Foxp1 misexpression in ESC-derived MNs produces functionally mature MNs with enhanced capacity to innervate limb muscles in vitro.

Transplanted Foxp1-programmed MNs innervate limb muscles. Hb9::GFP MNs have been demonstrated to preferentially project axons towards axial muscle on transplantation into developing chick embryos ${ }^{6}$. A more recent study found that spontaneously differentiated mouse ESC-derived MNs could project axons towards limb muscles on transplantation, but did not show whether they innervated and formed synapses with the limb muscles ${ }^{19}$. We injected GFP ${ }^{+}$EBs generated from Hb9::GFP and Hb9::Foxp1 ESCs into the neural tube of st.14-17 chick embryos at brachial regions and collected the embryos 3-5 days later (Fig. 7a). First, we confirmed that the molecular profile of the MNs did not change on transplantation. The majority of transplanted Hb9::GFP ESC-derived MNs expressed Lhx3 and Hoxa5, despite injection into Hoxc8 ${ }^{+}$brachial regions of the chick neural tube (Supplementary Fig. 6a,b). In contrast, transplanted Hb9::Foxp1 MNs contained a mixture of $\mathrm{Lhx}^{+}$ $(35 \pm 6 \%), \quad L h x 1^{+} / \mathrm{Foxp}^{+}(30.6 \pm 0.1 \%)$ and Foxp ${ }^{+}$only $(36.6 \pm 1.8 \%)$ cells, which matched the distribution of MN subtypes characterized in vitro (Supplementary Fig. 6a,b). Transplanted Hb9::GFP MNs that migrated into the ventral horn of the spinal cord typically settled in a medial position where endogenous MMC MNs are located, while transplanted Hb9::Foxp1 MNs migrated more laterally towards endogenous LMC MNs (Fig. 7b).

We first analysed axon projections 3 days post transplantation, when both endogenous and transplanted MN axons have reached their target muscles (Fig. 7c). Control Hb9::GFP transplants contained more $\mathrm{GFP}^{+}$axons projecting dorsally towards axial muscles than laterally towards limb muscles (mean \pm s.e.m. $=$ $57.3 \pm 3.6 \%$ axial versus $42.7 \pm 3.6 \%$ limb). By comparison, Hb9::Foxp1 transplants showed significantly more GFP ${ }^{+}$axons projecting laterally towards limb muscles (mean \pm s.e.m. $=$ $40.2 \pm 4.7 \%$ axial versus $59.8 \pm 4.7 \%$ limb) (Fig. $7 \mathrm{~d}$ ). At later stages in development, neural circuit refinement occurs as inappropriate or non-functional connections are removed by axon pruning and programmed $\mathrm{MN}$ cell death ${ }^{38}$. To see whether increased muscle specificity of transplanted ESC-derived MNs could be observed during this process, we analysed embryos 4 days after transplantation. $100 \%(8 / 8)$ of control Hb9::GFP transplants maintained their axial muscle-innervating 
a
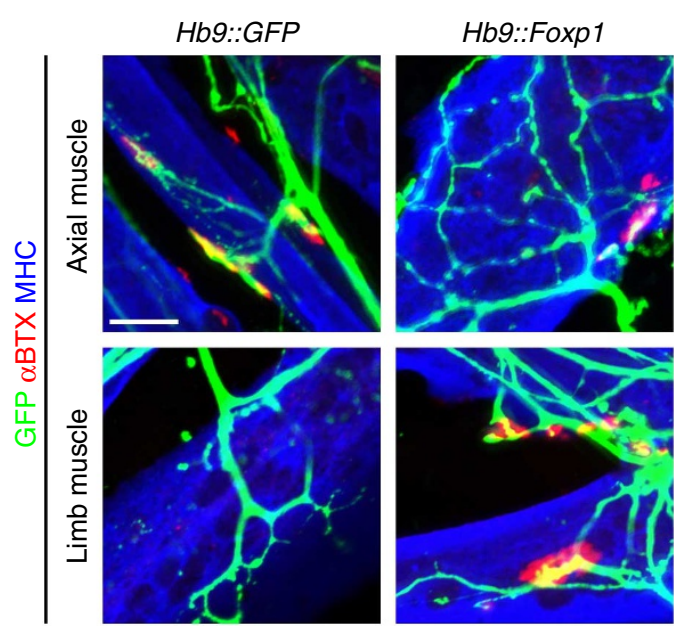

d

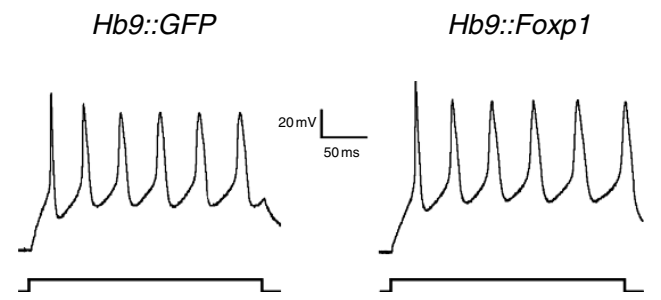

b

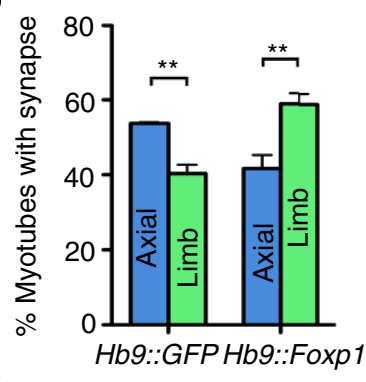

c

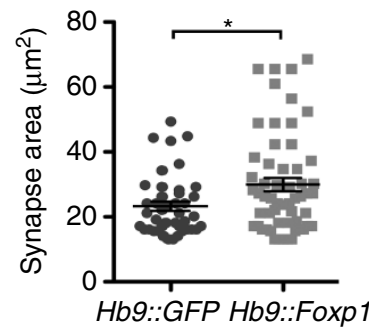

e

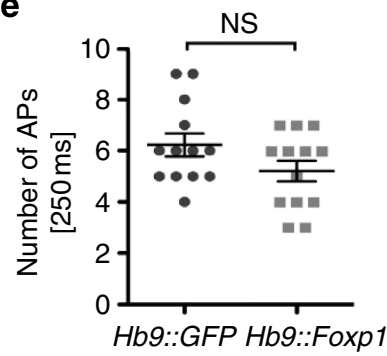

Figure 6 | Hb9:\#Foxp1 MNs preferentially form synapses with limb muscles. (a) Hb9::GFP MNs formed neuromuscular junctions (GFP $+/ \alpha B T X+$ ) with axial myotubes more frequently than with limb myotubes. By contrast, Hb9::Foxp1 MNs formed synapses with limb-derived myotubes more frequently than with axial myotubes. Scale bar, $20 \mu \mathrm{m}$. (b) Quantification of the frequency of synapse formation with primary myotubes in vitro. The percentage of myotubes with $\mathrm{GFP}^{+} / \alpha \mathrm{BTX}^{+}$synapses over the total number of myotubes with $\mathrm{GFP}^{+}$axon contacts was calculated for each cell line/muscle pairing (mean \pm s.e.m.; $n=3$ independent experiments, 20 images per experiment; Student's $t$-test). ${ }^{\star \star} P=0.0046$ for Hb9::GFP and ${ }^{\star \star} P=0.0081$ for Hb9::Foxp1. (c) Hb9::Foxp1 MNs formed significantly larger neuromuscular junctions with limb muscle than Hb9::GFP MNs, as determined by measuring the area of $\mathrm{GFP}^{+} / \alpha \mathrm{BTX}^{+}$synapses (mean \pm s.e.m.; $n=5$ images per cell line, 117 total synapses; Student's $t$-test). ${ }^{\star} P=0.0143$. (d) Representative recordings of Hb9::GFP and Hb9::Foxp1 ESC-derived MNs. Both cell types responded to a $+0.1 \mathrm{nA}$ long duration (250 ms) current pulse with multiple action potentials. (e) Quantification of the number of action potentials (APs) fired by ESC-derived MNs during a $+0.1 \mathrm{nA}, 250 \mathrm{~ms}$ current pulse (mean \pm s.e.m.; $n=13$ cells per cell line; Student's $t$-test). NS, not significant, $P>0.5$.

projections, but none $(0 / 8)$ contained $\mathrm{GFP}^{+}$projections into the forelimbs (Fig. 7c,e). 75\% (6/8) of Hb9::Foxp1 transplants had $\mathrm{GFP}^{+}$axial projections, which is not unexpected since $35 \%$ of transplanted Hb9::Foxp1 MNs expressed Lhx3. However, 50\% (4/ 8) of Hb9::Foxp1 transplants also contained $\mathrm{GFP}^{+}$limb projections (Fig. $7 \mathrm{c}, \mathrm{e})$. Of these limb projections, the majority of axons $(65 \%)$ innervated the distal dorsal limb, likely due to their robust expression of EphA4, c-Ret, and Lhx1, with the remainder extending ventrally.

To determine whether Foxp1 ${ }^{+}$ESC-derived MNs innervate other types of muscle at non-limb levels of the embryo, we transplanted Hb9::Foxp1 ESC-derived MNs into the thoracic chick neural tube and analysed axon projections 3 and 4 days later. After 3 days, the majority of embryos contained GFP ${ }^{+}$ axon projections to both axial and intercostal muscles, but not the sympathetic ganglia (Supplementary Fig. 6c). One day later, we found that $60 \%(3 / 5)$ of embryos had GFP ${ }^{+}$axial projections, but $0 \%(0 / 5)$ had distal projections to the intercostals (Supplementary Fig. $6 \mathrm{c}, \mathrm{d}$ ). These results indicate that Hb9::Foxp1 ESC-derived MNs failed to stably innervate intercostal muscles, and likely underwent axonal dieback and/or cell death.

We next asked if transplanted Hb9::Foxp1 ESC-derived MNs projected to both axial and limb muscles due to their mixed composition of MMC and LMC MNs. To address this, we injected dextran into either the axial or limb nerve branches of transplanted chick embryos, and analysed the molecular profile of the retrogradely labelled ESC-derived MNs. When dextran was injected into the axial nerve, $100 \%$ of labelled $H b 9:: G F P$ and Hb9::Foxp1 ESC-derived MNs expressed Lhx3 and 0\% expressed Foxp1 (Supplementary Fig. 7a). In contrast, when dextran was injected into the limb nerve, 0\% of labelled Hb9::Foxp1 ESCderived MNs expressed Lhx 3 and $88.9 \pm 11.1 \%$ expressed Foxp1 (Supplementary Fig. 7b). Hb9::GFP ESC-derived MNs were not labelled after limb nerve injections, in agreement with their failure to innervate limb muscles in vivo. These results confirm that Hb9::Foxp1 ESC-derived MNs innervated muscles appropriate for their MMC and LMC molecular identities.

We lastly analysed transplanted ESC-derived MNs for presence of synaptic proteins and formation of neuromuscular junctions in vivo (Fig. 8). At Hamburger-Hamilton ( $\mathrm{HH}$ ) stages 30-32, transplanted Hb9::GFP axons innervated axial muscles, demonstrated by presence of $\mathrm{GFP}^{+}$boutons that contained the presynaptic protein, Synaptotagmin 2 (Fig. 8a). These boutons overlapped with a high number of postsynaptic acetylcholine receptors on chick axial muscles (marked by punctate labelling of Alexa ${ }^{647}$-conjugated $\left.\alpha \mathrm{BTX}\right)$, suggesting formation of 
a

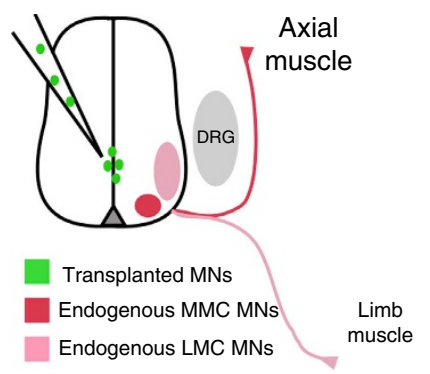

b

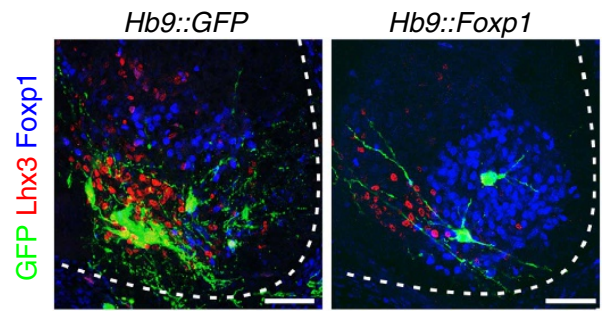

C

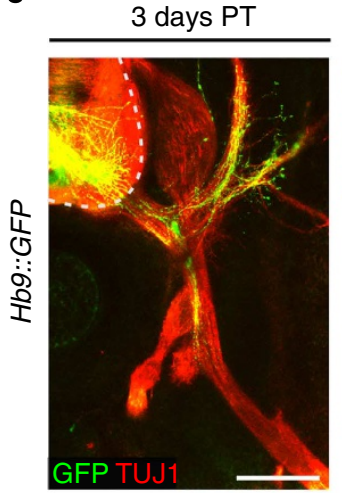

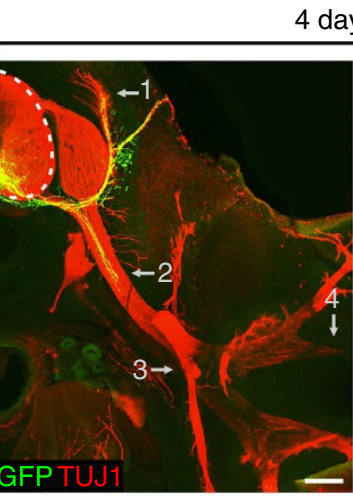

4 days $\mathrm{PT}$
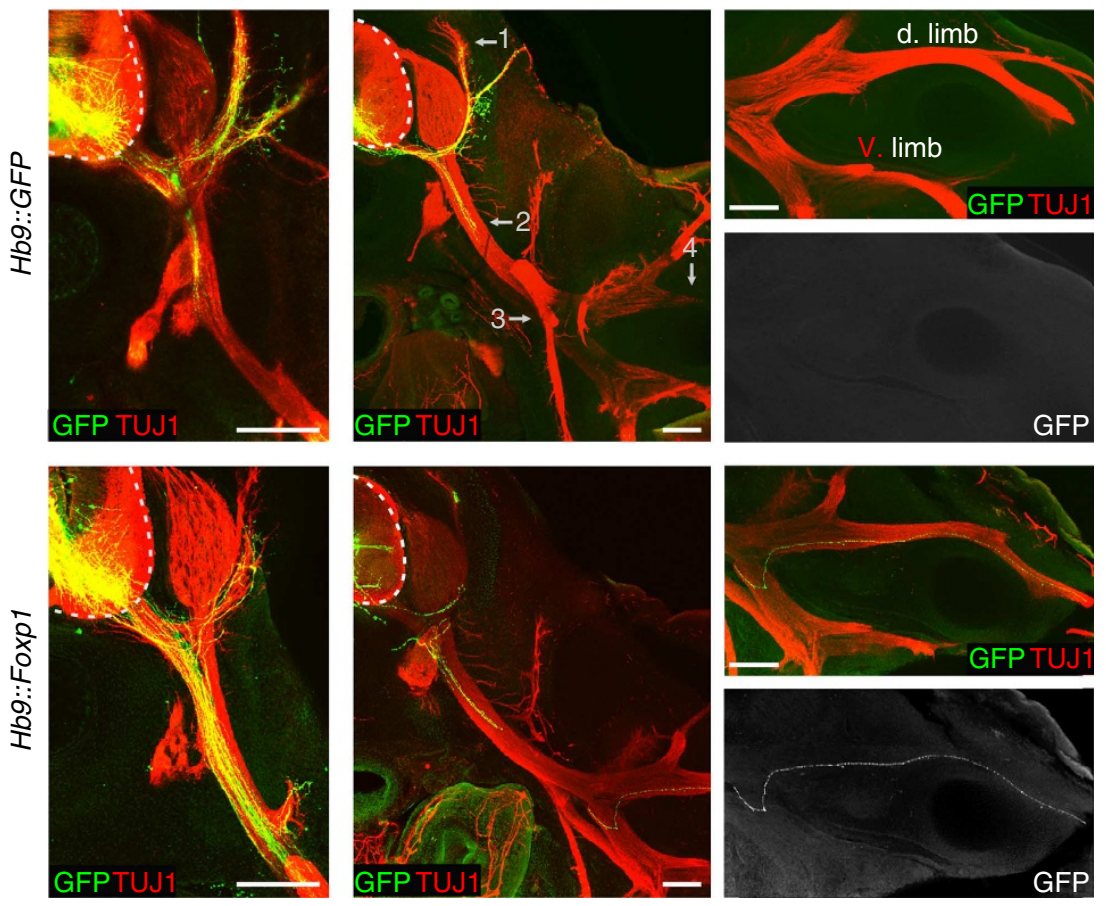

d

e
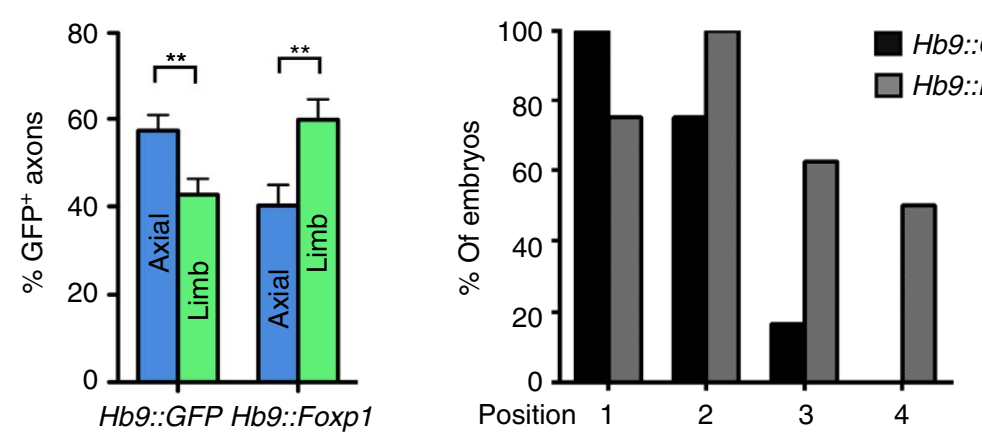

Figure 7 | Hb9:-Foxp1 MNs innervate distal limb muscles in vivo. (a) Transplantation scheme. DRG, dorsal root ganglion. (b) Transverse sections of the ventral horn chick spinal cords showing GFP ${ }^{+}$transplanted MNs. Hb9::GFP MNs settled at a medial position associated with endogenous Lhx3+ MMC MNs. Hb9::Foxp1 MNs settled at lateral positions associated with endogenous Foxp1 ${ }^{+}$LMC MNs. Scale bars, $50 \mu$ m. (c) Transverse vibratome sections of transplantations. Hb9::GFP ESC-derived MNs projected axons towards dorsal axial muscles both 3 and 4 days post transplantation (PT). Hb9::GFP axons that projected laterally did not extend into the limb. The majority of Hb9::Foxp1 ESC-derived MNs projected axons laterally towards the limbs 3 days PT and limb projections were maintained at 4 days PT. 'd. limb' $=$ dorsal limb. 'v. limb' $=$ ventral limb. 'DRG' $=$ dorsal root ganglion. Scale bars, $200 \mu m$.

(d) Quantification of the percentage of transplanted GFP + MN axons that projected towards axial and limb muscles at 3 days PT (mean \pm s.e.m.; $n=10$ transplants per cell line; Student's $t$-test). ${ }^{\star \star} P=0.008$. (e) Quantification of transplanted GFP ${ }^{+}$axons at 4 days PT. Graph shows the percentage of chick embryos that had $\mathrm{GFP}^{+}$axons at positions 1-4. Position $1=$ axial muscle. Position $2=$ halfway to brachial plexus. Position $3=$ brachial plexus. Position $4=$ distal limb (these are marked in $\mathbf{c}$ ). $n=8$ transplants per cell line. 
neuromuscular junctions. In contrast, Hb9::Foxp1 axons that innervated axial muscles showed less co-localization of Synaptotagmin 2 and $\alpha$ BTX, indicating fewer synapses (Fig. 8a). To quantify this difference, we counted the number of Synaptotagmin $2^{+} / \alpha \mathrm{BTX}^{+}$synapses normalized to the number of $\mathrm{GFP}^{+}$ axons, which showed that $H b 9:: G F P$ MNs formed significantly more synapses with axial muscle than Hb9::Foxp1 MNs (Fig. 8c,d). Transplanted Hb9::Foxp1 MNs by contrast produced many Synaptotagmin 2 and $\alpha \mathrm{BTX}$ clusters at the terminals of $\mathrm{GFP}^{+}$axons in dorsal limb regions, a result that was never seen with Hb9::GFP MNs (Fig. 8b,d). Finally, using an antibody known to label premotor synaptic terminals in the chick spinal cord ${ }^{39}$, we found bright Synaptotagmin ${ }^{+}$puncta clustered around the cell bodies and dendrites of both Hb9::GFP and Hb9::Foxp1 MNs (Fig. 8e), suggesting that these transplanted cells are capable of receiving presynaptic inputs from the host spinal cord. Collectively, these data show that Foxp1 misexpression during ESC MN differentiation generates bona fide LMC MNs that successfully integrate into the spinal neural circuitry and innervate distal limb muscles in vivo, unlike control ESCderived MNs.

\section{Discussion}

MNs are essential for all voluntary and involuntary muscle movements as well as visceral organ functions, illustrated by the catastrophic and often fatal loss of motor control associated with $\mathrm{MN}$ disease ${ }^{8}$. Considerable effort has been accordingly directed towards generating MNs from stem cells for use in modelling MN disease pathologies, and potentially as cellular therapies ${ }^{1-5}$. In most stem cell studies, little attention is given to the fact that MNs in vivo are not a single cell type, but rather a collection of functionally distinct neurons. In the developing spinal cord, MNs are segregated into topographically organized columns and pools along rostrocaudal, mediolateral and dorsoventral axes, largely controlled by the expression of unique transcription factor combinations (Fig. 9a ${ }^{7-13}$. This organization allows $\mathrm{MNs}$ to correctly innervate different body muscles and receive appropriate presynaptic inputs from sensory neurons, spinal interneurons and descending pathways needed to form stable and functional motor circuits $^{40}$. In agreement with previous reports ${ }^{6,19}$, we have found that differentiation of mouse and human ESCs with RA and SAG, a protocol that is widely used in MN differentiation studies, predominantly produces MMC MNs that preferentially innervate and form synapses with axial but not limb muscles (Fig. 9b). Our results show that the molecular and functional diversity of these stem cell-derived MNs can be expanded through the programmed expression of Foxp1, which transforms newly born MNs towards an LMC fate (Fig. 9b). Importantly, Hb9::Foxp1 ESC-derived MNs innervate and form synapses with distal limb muscles-the defining property of LMC MNs.

One of the current bottlenecks to achieving $\mathrm{MN}$ diversity in vitro is our limited understanding of how this process occurs in vivo, particularly the nature of environmental signals involved. Removal of exogenous growth factors has been shown to broaden the types of MNs generated in vitro, but this approach diminishes MN production efficiency to $\sim 8 \%$ of total cells ${ }^{19}$. About $\sim 38 \%$ of spontaneously generated ESC-derived MNs express Foxp1 (that is, $\sim 3 \%$ of the total cells), and only a portion of these cells express LMC motor pool markers such as Etv4 and Pou3f1 $\left(\sim 18 \% \text { and } \sim 9 \% \text { of Foxp } 1^{+} \text {MNs, respectively }\right)^{19}$. Moreover, few $\mathrm{Lhxl}^{+} \mathrm{LMCl} \mathrm{MNs}$ are generated under these conditions even after the addition of RA ( $\sim 15 \%$ of Foxp ${ }^{+}$MNs, that is, $<0.5 \%$ of total cells $)^{19}$. This markedly contrasts with our results, where $30-40 \%$ of the total cells become MNs, over half of which express high levels of Foxp1 and adopt an LMC identity (that is, $15-20 \%$ of total cells). It was recently observed that addition of two types of Shh agonists (Purmorphamine and SAG) in addition to RA, results in accelerated generation of Foxp $1^{+} \mathrm{MNs}$ from human pluripotent stem cells 4 . $\sim$ About $30 \%$ of the cells become MNs, of which $\sim 65 \%$ express Foxp1 (that is, $\sim 20 \%$ of total cells). Interestingly, only $1-5 \%$ of these Foxp $1^{+}$MNs express Lhx1 (ref. 41). It remains to be seen whether these hESC-derived MNs also express LMC motor pool markers and preferentially innervate limb muscles.

It is notable that both of these previously described protocols for generating $\mathrm{Foxp}^{+}$ESC-derived MNs favour LMCm production. This contrasts with Hb9::Foxp1 ESCs, which preferentially form $\mathrm{LMCl}$ rather than $\mathrm{LMCm} \mathrm{MNs}$, reflected in high levels of Lhx1, Etv4, EphA4 and c-Ret expression. As many as $40 \%$ of Hb9::Foxp1 MNs expressed Lhx1, and 32\% of total MNs expressed Etv4-both significant increases over previous methods. These MNs also displayed dramatically altered axon projections in vitro and in vivo, with both assays showing a preference for dorsal limb muscle over axial muscle. LMCl MNs were also better restored to wild-type levels than LMCm or HMC MNs in Hb9::Foxp1; Foxp1-1- embryos. While the bias seen in vitro could be partially explained by the inclusion of RA in the $\mathrm{MN}$ differentiation procedure, as RA is known to promote $\mathrm{LMCl}$ formation $^{22}$, it doesn't easily account for the bias seen in vivo. Another factor that could potentially influence LMC subdivision is the differential activity of the $\mathrm{Hb} 9$ promoter used to drive ectopic Foxp1 expression in different MN subtypes. While Hb9 is expressed by all somatic MNs, it is notably elevated in $\mathrm{LMCl}$ MNs and low in $\mathrm{LMCm} \mathrm{MNs}{ }^{12,13,15,42,43}$. Consequently, $\mathrm{LMCl}$ cells may contain more ectopically expressed Foxp1 than their LMCm counterparts, which could increase the efficiency and/or completeness of their transformation.

Despite these predilections, Hb9::Foxp1 ESC-derived MNs still produced substantial numbers of $\mathrm{Foxp} 1^{+} / \mathrm{Lhx} 1^{-} / \mathrm{Pou}_{\mathrm{f} 1}{ }^{+}$ MNs, which likely represent an LMCm-associated motor pool ${ }^{25}$, and were also capable of innervating ventral limb muscles on transplantation. We further found that Foxp1 misexpression did not alter Hox protein expression, which agrees with previous reports of Foxp1 acting downstream or in combination with cervical Hox proteins to direct LMC motor pool formation in the embryonic spinal cord ${ }^{12,13,24}$. The signals required for the formation of most cervical LMC motor pools remain unknown; however, seeing as Foxp1 $1^{-/-}$embryos lack all known LMC motor pool markers ${ }^{12,13}$, it seems likely that Foxp1 plays a central role in the assignment of pool identities. In this regard, Foxp1 overexpression during ESC MN differentiation could be a useful tool for identifying novel Foxp1 targets in spinal MNs.

In addition to using exogenous signalling molecules to direct the formation of MNs from pluripotent stem cells, others have begun using transcriptional programming approaches to directly generate MNs from ESCs ${ }^{44}$ and fibroblasts ${ }^{45}$. The types of MNs generated from these procedures remain unclear; however, they most likely represent MMC MNs because Lhx3 overexpression is required for $\mathrm{MN}$ conversion ${ }^{44,45}$. Interestingly, $\mathrm{MNs}$ directly converted from fibroblasts cluster separately from embryonic and ESC-derived MNs in microarray analyses ${ }^{45}$, suggesting that directly converted MNs retain some epigenetic differences related to their fibroblast origin. In our study, we present a hybrid approach incorporating both developmental signalling molecules and transcriptional programming to generate LMC MNs efficiently in vitro. The advantages of this method are that it maintains normal differentiation efficiencies, could be applied to both mouse and human systems, and can be adapted to produce other types of MNs, such as thoracic and lumbar populations, as long as candidate transcription factors are known. In principle, it should be possible to combine the approach of misexpressing Foxp1 in combination with different Hox proteins to create PGC 
a
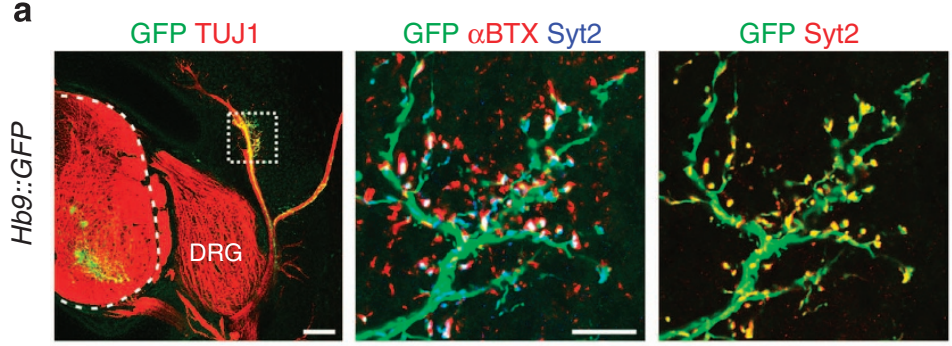

Syt2 $\alpha \mathrm{BT} X$
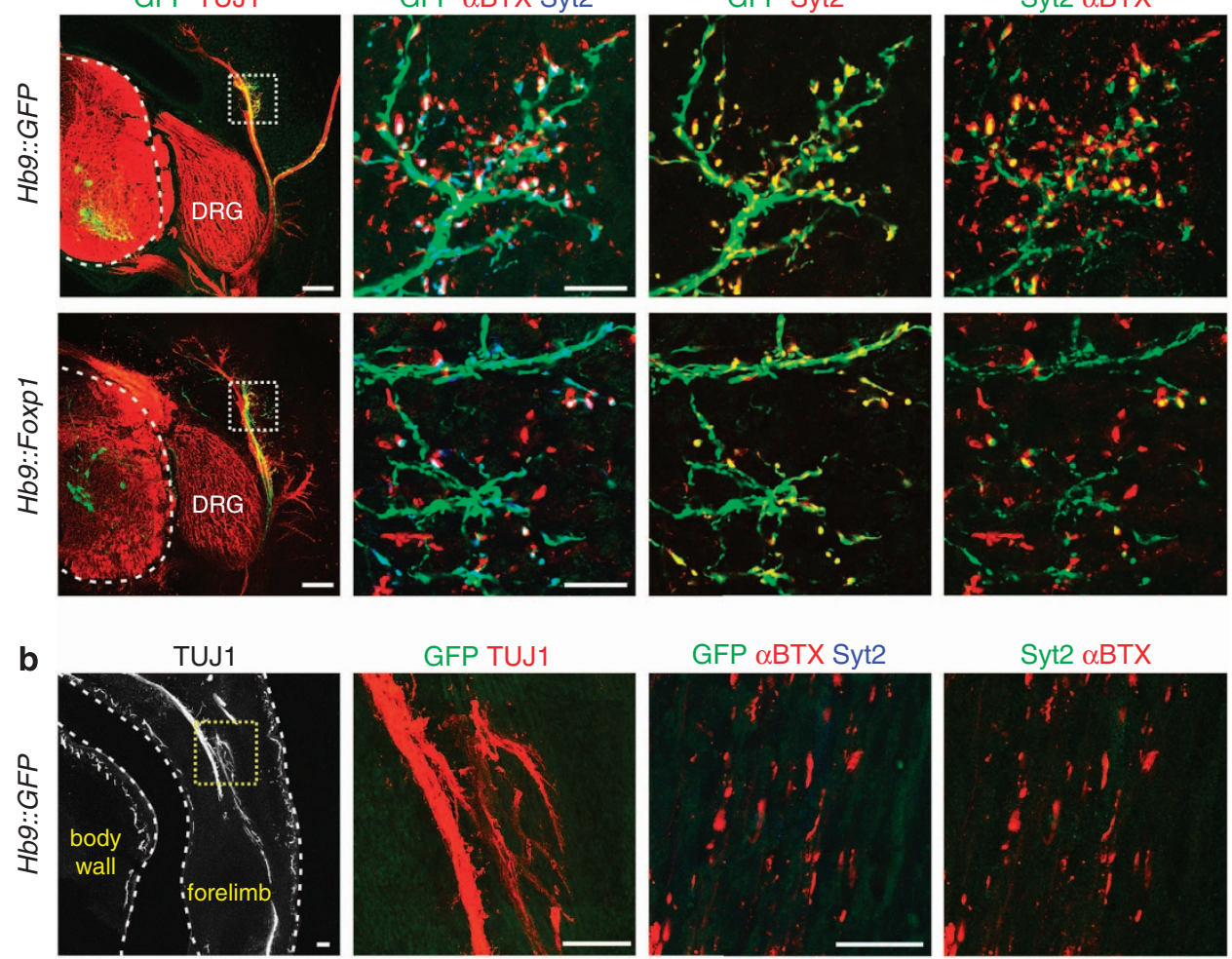

GFP TUJ1

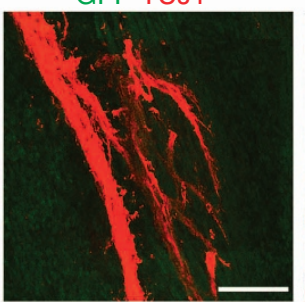

GFP $\alpha$ BTX Syt2
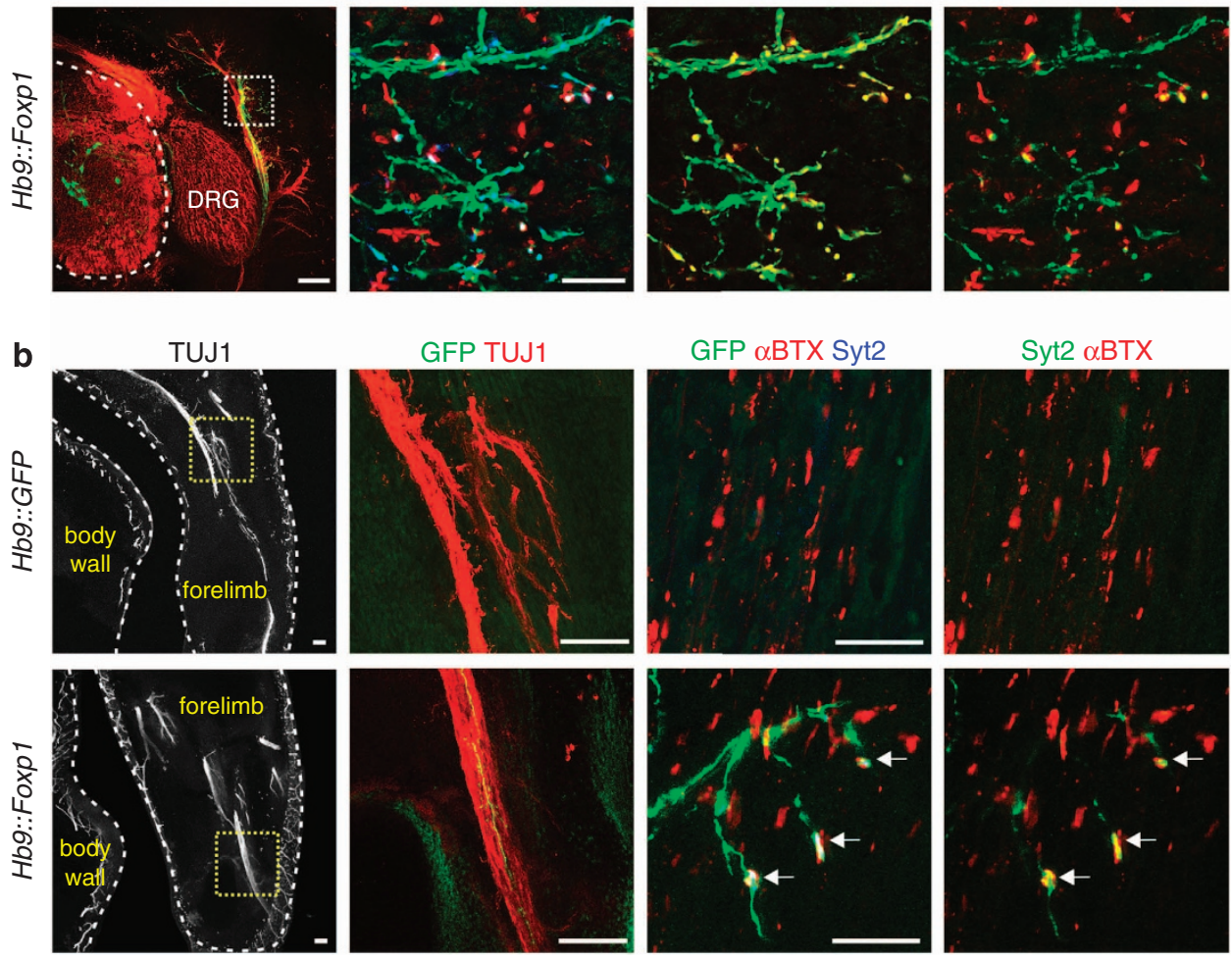

Syt2 $\alpha \mathrm{BTX}$
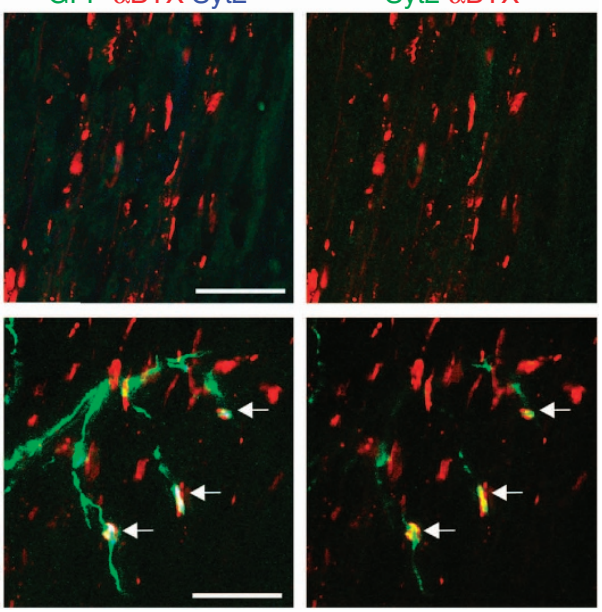

C

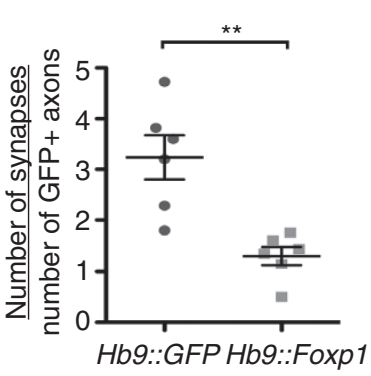

e
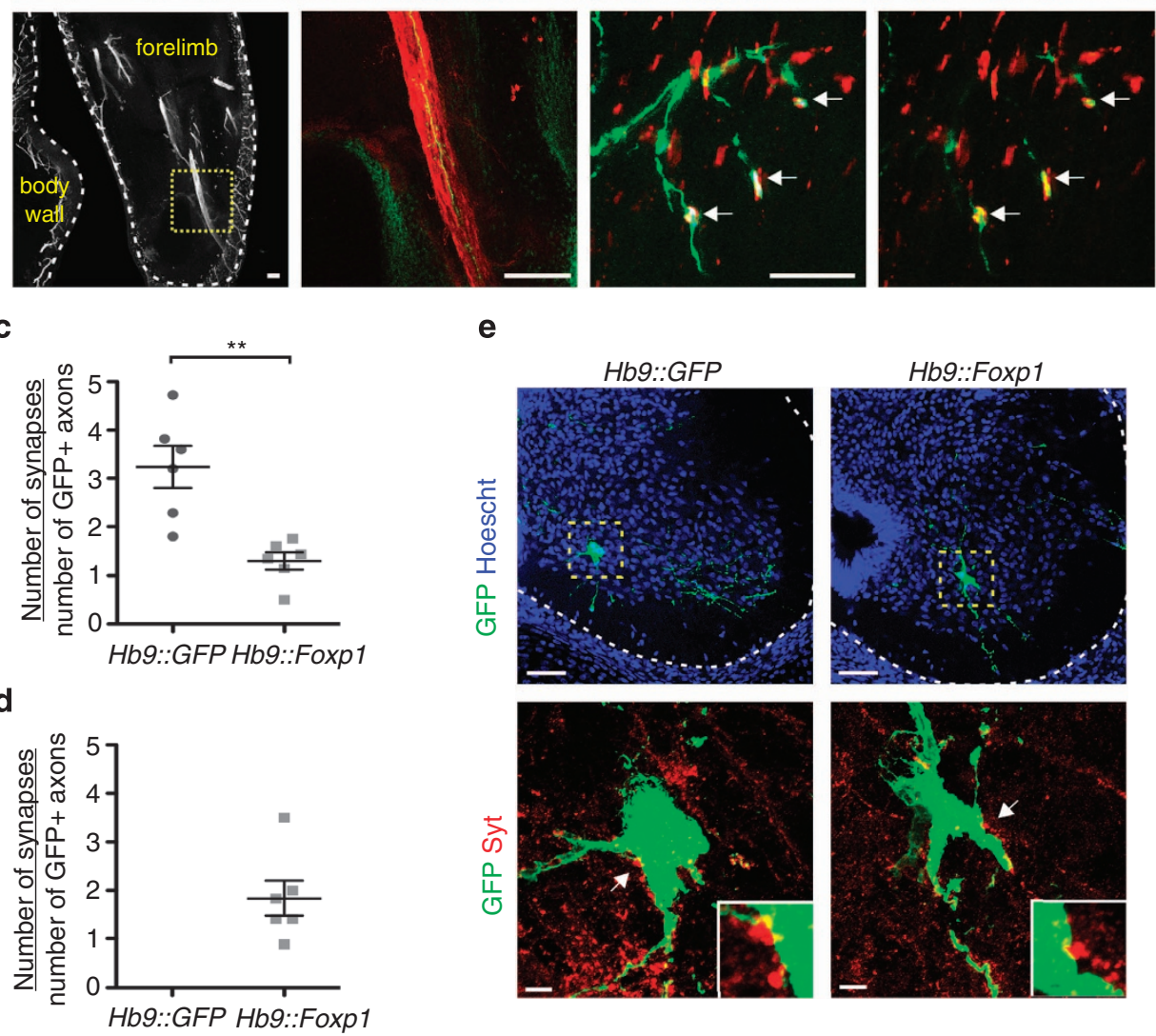

and lumbar LMC MNs from ESCs, which have never been effectively generated in vitro.

Finally, our studies stress the importance of knowing what type of MNs are being produced within the stem cell cultures for the purposes of disease modelling and cellular therapies. Limbinnervating MNs are thought to be among the earliest affected in

ALS, while MMC and HMC MNs are principally affected in spinal muscular atrophy ${ }^{8,46}$. It is thus possible that Hb9::Foxp1 ESC-derived MNs might exhibit enhanced sensitivity to ALSassociated mutations, compared with the MMC MNs typically produced from other ESCs. These differences in MN identity are also paramount for the successful use of stem cell-derived MNs in 
regenerative contexts. Our results show that different classes of ESC-derived MNs exhibit distinct axon growth potentials and preferences in forming synaptic contacts. It is notable that transplanted Hb9::Foxp1 ESC-derived MNs successfully innervated and formed synapses with limb muscles in vivo, an activity that was not seen with control Hb9::GFP MNs. These latter cells were nevertheless capable of innervating axial muscles consistent with their MMC identity. These results are reminiscent of experiments in which thoracic spinal cord segments were transplanted into lumbar levels of chick embryos. The transplanted thoracic MNs initially innervated hindlimb muscles, but at later ages, both MNs and muscles degenerated resulting in impaired hindlimb motor functions ${ }^{47,48}$. Together, these studies suggest that correct matching of MN and muscle subtypes may be critical for optimal synapse formation and function. This detail is incredibly important in the context of MN diseases-both for modelling disease pathology in vitro with $\mathrm{MN}$-muscle co-cultures and for transplantation into the spinal cord to replace damaged cells. It is very likely that generating specific MN subtypes from stem cells and matching them with appropriate and inappropriate muscle types will reveal novel and significant discoveries into MN development and neurodegenerative disease pathologies.

\section{Methods}

Mouse husbandry. Hb9::GFP, Hb9::Foxp1, and Foxp $1^{+/-}$heterozygous mice were maintained as previously described ${ }^{13,15,49}$. Hb9::Foxp1 heterozygous mice were crossed with Foxp1 $1^{+/}$mice to generate Hb9::Foxp1; Foxp1 ${ }^{+/}-$mice. These mice were then interbred with Foxp $1^{+/-}$mice to produce Hb9::Foxp1; Foxp $1^{-1-}$ embryos. The Hb9::Foxp1 transgene contains an IRES-GFP sequence but GFP expression levels were very low in ESC-derived MNs. All mice were maintained and tissue were collected in accordance with guidelines set forth by the UCLA Institutional Animal Care and Use Committee.

Hb9:FFoxp1 mouse ESC line generation and characterization. Superovulation was induced in $H b 9:: G F P$ heterozygous female mice by sequential injections of pregnant mare serum gonadotropin (5 IU) followed by human chorionic gonadotropin (50 IU) $48 \mathrm{~h}$ later per female. These mice were paired overnight with Hb9::Foxp 1 transgenic male mice. E0.5 pre-implantation embryos were isolated the next morning and incubated in hyaluronidase $\left(0.3 \mathrm{mg} \mathrm{ml}^{-1}\right.$; Sigma) until cumulus cells were removed from the embryos. About 24 embryos were collected in total and cultured in KSOM media (Millipore) until the blastocyst stage ${ }^{50}$. About 15 out of 24 embryos developed into blastocysts and were plated on mitomycin-treated mouse embryonic fibroblasts (MEFs) in mouse ESC media (Dulbecco's Modified Eagle's Medium/ (DMEM) high glucose, 10\% ES-qualified FBS, 1\% penicillin/ streptomycin, $1 \%$ glutamax, $1 \%$ nonessential amino acids, Primocin $\left(50 \mu \mathrm{g} \mathrm{ml}^{-1}\right.$; Invivogen), 2-mercaptoethanol $(560 \mathrm{nM})$ and Leukaemia Inhibitory Factor (ESGRO; Millipore)) plus MEK1 inhibitor (50 $\mu \mathrm{M}$; PD98059; Cell Signaling Technologies). Except as noted, media components were obtained from Invitrogen. About 4 days after plating, 12 blastocysts had hatched and attached to MEFs. Attached inner cell masses were monitored and passaged after 3-4 days of growth.

Figure 8 | Transplanted ESC-derived MNs form synapses with correct muscles. Analysis of synapse formation 5 days after transplantation of Hb9::GFP and Hb9::Foxp1 ESC-derived MNs. Scale bars, $100 \mu \mathrm{m}$ for TUJ1 images and $20 \mu \mathrm{m}$ for $\alpha$ BTX images. (a) Transplanted GFP ${ }^{+}$axons innervating axial muscle. Hb9::GFP axons co-localized with a high density of presynaptic (mouse specific Synaptotagmin 2 (Syt2)) and postsynaptic ( $\alpha$ BTX) markers. Hb9::Foxp1 axons displayed fewer synaptic boutons. Boxes represent the areas imaged for synapse markers. DRG, dorsal root ganglion. (b) Transplanted GFP ${ }^{+}$axons innervating limb muscle. Hb9::GFP ESC-derived MNs did not innervate and form synapses with limb muscle. Hb9::Foxp1 axons in the distal limb co-localized with Synaptotagmin 2 and $\alpha$ BTX (arrows). Yellow boxes represent the areas shown in GFP/TUJ1 images where GFP ${ }^{+}$axons were analysed for synapse markers. (c) Quantification of the number of synapses formed by each cell line with axial muscle in vivo (mean \pm s.e.m.; $n=6$ images per cell line; Student's $t$-test). ${ }^{\star} P=0.002$. (d) Quantification of the number of synapses formed by each cell line with limb muscles in vivo (mean \pm s.e.m.; $n=6$ images per cell line; Student's t-test). Hb9::GFP control cells showed no synapses in this assay. (e) Transplanted ESC-derived MNs in the ventral horns of chick spinal cords (outlined in boxes) had bright Synaptotagmin (Syt) ${ }^{+}$punctae surrounding their cell body and dendrites. Scale bars, $50 \mu \mathrm{m}$ in top row and $5 \mu \mathrm{m}$ in bottom row. Arrows point to regions shown in insets.

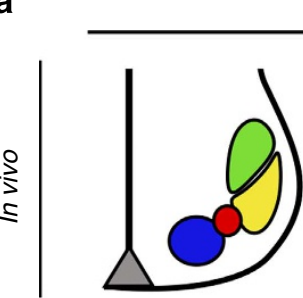

MN columns

b

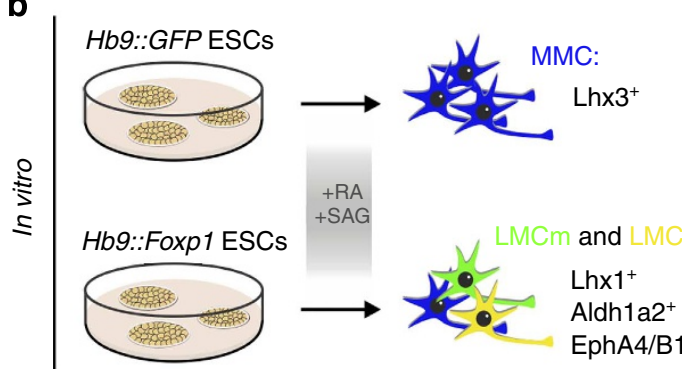

HMC: Hb9, Isl1

MMC: Hb9, Isl1, Lhx3
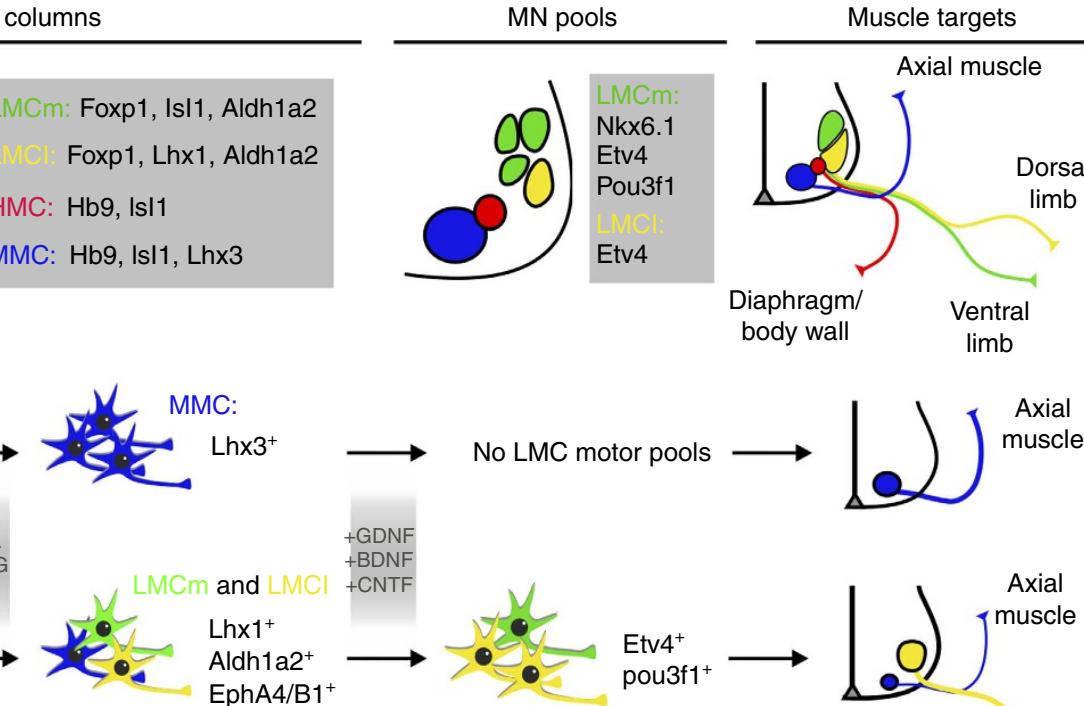

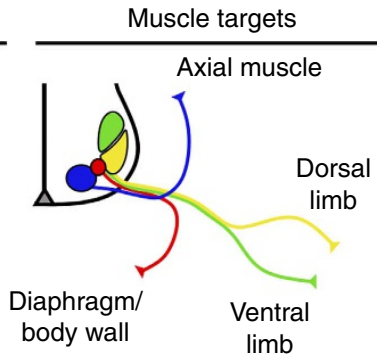

Axial muscle

Figure 9 | Summary of spinal MN diversity in vivo and from ESCs. (a) At cervical levels in the developing spinal cord, MNs are segregated into different columns that express different transcription factors and proteins. These columns are further organized into motor pools, or groups of MNs that innervate the same muscle. These different MN subtypes innervate and form synapses with distinct muscles. (b) Under standard differentiation conditions with retinoic acid (RA) and smoothened agonist (SAG), the majority of control Hb9::GFP ESC-derived MNs expressed Lhx3-a marker of MMC MNs, and did not express markers of LMC MNs. Hb9::GFP ESC-derived MNs preferentially projected axons towards and formed synapses with axial muscle both in vitro and in vivo. Conversely, Foxp1 misexpression resulted in large numbers of bona fide LMC MNs that expressed multiple LMCm and LMCI molecular markers and preferentially innervated and formed synapses with limb muscle both in vitro and in vivo. 
MEK1 inhibitor was removed from the media after the appearance of ES colonies. Mouse ESC colonies appeared within 1 week and were expanded on MEFs until confluent $^{51}$. Ten final mouse ESC lines were genotyped for presence of the two transgenes. Four lines contained both the Hb9::Foxp1 and Hb9::GFP transgenes and three lines contained only the Hb9::GFP transgene. All mouse ESCs were maintained in mouse ESC media on MEFs.

Mouse ESC differentiation to MNs. HBG3 Hb9::EGFP mouse ESCs (a gift from $\mathrm{H}$. Wichterle, Columbia University) and newly generated Hb9::GFP and Hb9::Foxp 1; Hb9::GFP mouse ESCs were maintained and differentiated into MNs as previously described ${ }^{16,52}$. Briefly, mouse ESCs were first plated on gelatin to remove MEFs prior to differentiation, and then plated in $60 \mathrm{~mm}$ bacterial Petri dishes in core MN medium to induce EB formation. Core MN medium consisted of a 1:1 mixture of DMEM/F12 and Neurobasal Medium supplemented with 10\% knockout serum replacement, 1\% Glutamax, 2-mercaptoethanol $(560 \mathrm{nM}), 1 \%$ penicillin/streptomycin and Primocin. Except as noted, media components were obtained from Invitrogen. About 2 days later, N2 supplement ( $1 \times$, Invitrogen), RA ( $1 \mu \mathrm{M}$; Sigma), and SAG ( $1 \mu \mathrm{M}$; Calbiochem) were added to the EBs. After 5-6 days, the EBs were either dissociated and plated on matrigel-coated coverslips for immunostaining or used for transplantation and muscle assays. For culturing the MNs past day 6, RA and SAG were removed from the medium and replaced with GDNF (10 $\mathrm{n} \mathrm{ml}^{-1}$; Peprotech), Brain-Derived Neurotrophic Factor $\left(10 \mathrm{ng} \mathrm{ml}^{-1}\right.$; Peprotech), and Ciliary Neurotrophic Factor (10 $\mathrm{ng} \mathrm{ml}^{-1}$; Peprotech).

Cell sorting of stem cell-derived MNs and RNA isolation. $H b 9:: G F P$ and Hb9::Foxp1 EBs were collected 5-7 days after RA and SAG addition. EBs were dissociated using ice-cold Trypsin-EDTA (0.25\%; Invitrogen) for $6 \mathrm{~min}$ at room temperature, followed by trituration in L-15 Leibovitz media (Thermo Scientific) ${ }^{53}$. The dissociated cells were pipetted through $40 \mu \mathrm{m}$ cell strainers and live GFP ${ }^{+}$ MNs (identified as lacking staining for 7AAD) were sorted and collected using a BD ARIA II FACS machine in the UCLA Broad Stem cell center core facility. Total RNA was isolated from $\mathrm{GFP}^{+}$MNs using an RNAeasy MiniKit (Qiagen).

Quantitative PCR. Quantitative PCR analysis of MN populations was performed as previously described ${ }^{21}$. The following primer pairs were used: mouse Foxp1 $3^{\prime} U T R$, forward $5^{\prime}$-cagccacgaaagaaacagaag- $3^{\prime}$ and reverse $5^{\prime}$-ggtcctggtcacctgattata$3^{\prime}$; mouse Aldh 1a2, forward $5^{\prime}$-catggtatcctccgcaatg- $3^{\prime}$ and reverse $5^{\prime}$-gcgcatttaaggcat tgtaac- $3^{\prime}$; mouse EphA4, forward $5^{\prime}$-ctgagccagtaccgaataga- $3^{\prime}$ and reverse $5^{\prime}$-gagggc gaagacaaagtaaa- $3^{\prime}$; mouse $E p h B 1$, forward $5^{\prime}$-gaagaaaggaaggaggtggaa- $3^{\prime}$ and reverse $5^{\prime}$-agggaggagtaagaaaggagaa- $3^{\prime}$; mouse $c$-Ret, forward $5^{\prime}$-ctgactgaggtgagagaacaag-3' and reverse $5^{\prime}$-acacgtaccatagcagcataaa- $3^{\prime}$; mouse $c$-Met, forward $5^{\prime}$-tcagccatcccaa tgttctc- $3^{\prime}$ and reverse $5^{\prime}$-cgcagatctccatgcttcata- $3^{\prime}$; mouse Fgfrl, forward $5^{\prime}$-aggttag ccagagggtagaa- $3^{\prime}$ and reverse $5^{\prime}$-aggagaggaatatgaggagtagg $-3^{\prime}$; mouse $\beta$-Actin, forward $5^{\prime}$-gccaaccgtgaaaagatgac- $3^{\prime}$ and reverse $5^{\prime}$-gaggcatacagggacagcac $-3^{\prime}$. Relative mRNA expression levels were determined by normalizing crossing points for each gene to $\beta$-Actin and calculating fold changes of Foxp1 misexpression over control Hb9::GFP MNs.

Immunostaining. All embryos and cell cultures were fixed in $4 \%$ paraformaldehyde (Fisher). Antibody staining of cryosections and vibratome sections were performed as previously described ${ }^{13,21,54}$. Fixed adherent cell cultures were washed in block solution (PB, $1 \%$ heat-inactivated horse serum, $0.1 \%$ Triton X-100) for $10 \mathrm{~min}$, followed by incubation with primary antibodies overnight at $4^{\circ} \mathrm{C}$. The following primary antibodies were used: guinea pig anti-Aldh1a2 $(1: 20,000)^{55}$, goat anti-ChAT (1:200; Millipore AB144P) $)^{52}$, rabbit anti-EphA4 (1:500; Santa Cruz Sc-291) $)^{13}$, rabbit anti-Etv $4(1: 1,000)^{56}$, guinea pig anti-Foxp1 $(1: 16,000)^{13}$, sheep anti-GFP (1:800; AbD Serotec 4745-1051), rabbit anti-GFP (1:3,000; Life Technologies A6455) ${ }^{54}$, chick anti-GFP (1:1,000; Aves Labs GFP-1020), rabbit antiHb9 $(1: 8,000)^{42}$, guinea pig anti-Hoxa5 $(1: 8,000)^{25}$, goat anti-Hoxa9 $(1: 1,000$; Santa Cruz SC-17155) ${ }^{13}$, rabbit anti-Hoxc6 $(1: 1,000 \text {; Santa Cruz SC-66925) })^{13}$, mouse anti-Hoxc8 (1:2,000; Covance MMS-266R $)^{13}$, goat anti-Isl1 (1:8,000, R\&D Systems AF1837) ${ }^{13}$, mouse anti-Isl1/2 (39.4D5; 1:100; Developmental Studies Hybridoma Bank) ${ }^{57}$, mouse anti-Lhx1 (4F2; 1:100; Developmental Studies Hybridoma Bank) ${ }^{58}$, rabbit anti-Lhx3 (1:1,000; Abcam Ab14555), mouse anti-Lhx3 (67.4E12; 1:100; Developmental Studies Hybridoma Bank ${ }^{57}$, mouse anti-myosin heavy chain (MF20; 1:100; Developmental Studies Hybridoma Bank $)^{59}$, rabbit anti-Nkx6.1 $(1: 2,500)^{60}$; rabbit anti-nNOS $(1: 10,000 \text {; ImmunoStar } 24287)^{13}$, rabbit anti-Pou3f1 $(1: 2,000)^{61}$; mouse anti-Pou5f1 (1:1,000; Santa Cruz SC-5279), goat anti-Sox2 $\left(1: 2,000\right.$, Santa Cruz SC-17320) ${ }^{21,54}$, mouse anti-Synaptotagmin (mAb 30; 1:100; Developmental Studies Hybridoma Bank $)^{62}$, mouse anti-Synaptotagmin 2 (znp-1; 1:100; Developmental Studies Hybridoma Bank ${ }^{63,64}$, rabbit anti- $\beta$ III Tubulin (1:3,000; Covance MRB-435P) and mouse anti- $\beta$ III Tubulin (1:3,000; Covance MMS-435P). Monoclonal antibodies obtained from the DSHB were developed under the auspices of the NICHD and maintained by the University of Iowa, Department of Biology, Iowa City, IA 52242. Cells were washed and then incubated with Alexa ${ }^{488}$-, FITC-, Cy3-, Cy5- and DyLight ${ }^{649}$-conjugated secondary antibodies (Jackson Immunoresearch) for $1 \mathrm{~h}$ at room temperature the following day, and coverslips were mounted with Prolong Gold (Invitrogen). Representative images shown for antibody staining were successfully repeated at least 2-3 times.
Image analysis. DIC images were collected using a Zeiss Axioobserver microscope equipped with the Apotome optical imaging system. Fluorescent images were collected using either a Zeiss LSM5 Exciter or Zeiss LSM780 confocal imaging system. Images were processed using Adobe Photoshop and CorelDraw Software Cell number quantification was performed using the NIH ImageJ software suite with an ITCN add-in for automated cell counting.

Muscle and EB co-cultures. Hb9::GFP and Hb9::Foxp1 ESC-derived EBs with high levels of GFP expression were manually collected 5-6 days after RA/SAG addition. Axial, forelimb, dorsal hindlimb and ventral hindlimb muscles were dissected from HH stage 35 chick embryos. For adherent muscle co-cultures: dissected muscles were dissociated and plated onto matrigel-coated glass bottom dishes (MatTek Corporation). Hb9::GFP and Hb9::Foxp1 EBs were plated on top of the dissociated muscle and cultured for 3 days. Synapse formation was assayed using Alexa ${ }^{647}$-conjugated $\alpha$ BTX (1:300; Invitrogen). For three-dimensional collagen cultures, muscle explants were placed on either side of a single EB in a collagen bed (rat tail collagen IV, Calbiochem) ${ }^{65}$ and cultured for $24 \mathrm{~h}$. About $\sim 80 \%$ of muscle explants showed $>70 \%$ myosin heavy chain positive staining and $\sim 90 \%$ of explants showed axon growth. Data was extracted from this latter group. In both assays, muscle and $\mathrm{MN}$ co-cultures were incubated in core $\mathrm{MN}$ medium with $\mathrm{N} 2$ supplement and heparin $\left(10 \mu \mathrm{g} \mathrm{ml}^{-1}\right.$; Sigma).

Electrophysiology. Hb9::GFP and Hb9::Foxp1 EBs were dissociated using papain $\left(0.5 \mathrm{U} \mathrm{ml}^{-1}\right.$; Worthington) and plated at low density on Matrigel-coated $35 \mathrm{~mm}$ culture dishes $\left(1.2 \times 10^{4}\right.$ cells per dish) in core MN medium with neurotrophic factors and cultured for an additional 2-3 days. Recordings were made from $\mathrm{GFP}^{+}$ cells using standard whole-cell, current-clamp techniques at $20-22^{\circ} \mathrm{C}$. The recording bath solution contained: $\mathrm{NaCl}(120 \mathrm{mM}), \mathrm{KCl}(1.9 \mathrm{mM}), \mathrm{KH}_{2} \mathrm{PO}_{4}$ (1.2 mM), Na-bicarbonate $(20 \mathrm{mM}), \mathrm{CaCl}_{2}(2.2 \mathrm{mM}), \mathrm{MgCl}_{2}(1.4 \mathrm{mM})$, D-Glucose $(10 \mathrm{mM})$ and Hepes $(7.5 \mathrm{mM})$. Patch pipettes (resistance 3-5 M 2 ) were filled with internal recording solution containing: K-gluconate $(140 \mathrm{mM})$, Hepes $(10 \mathrm{mM})$, EGTA $(1 \mathrm{mM})$, Mg-ATP $(4 \mathrm{mM})$ and Na-GTP $(0.3 \mathrm{mM})$. The $\mathrm{pH}$ of both solutions was adjusted to 7.2 . Cells with resting potentials $<-30 \mathrm{mV}$ were discarded. Patched MNs (maintained in current-clamp mode at approximately $-70 \mathrm{mV}$ ) were stimulated with $0.1 \mathrm{nA}$ depolarizing current pulses of $250 \mathrm{~ms}$ duration. For each MN, this stimulus pulse was applied at least three times (with intervals $\geq 30$ s). Only data from cells in which the long stimulus pulse elicited the same number of APs were included in this study. Data were collected using Axopatch $2 \mathrm{~B}$ patch clamp amplifiers with 4-pole Bessel filtering at $5 \mathrm{kHz}$. Signals were digitized, stored and analysed using pClamp software (Axon Instruments). $\mathrm{AP}$ amplitude was measured as the voltage change from the threshold to the peak of the first AP in a train.

Chick transplantations. Fertilized chicken eggs (McIntyre Poultry and Fertile Eggs) were incubated at $38^{\circ} \mathrm{C}$ for $\sim 60 \mathrm{~h}$ to $\mathrm{HH}$ stage $14-17$ prior to use. Hb9::GFP and $H b 9::$ Foxp 1 ESC-derived MNs were transplanted into the lumen of the spinal cord, as described previously ${ }^{53}$. Briefly, GFP ${ }^{+}$EBs were manually collected 5-6 days after RA and SAG addition and cut into 4-6 pieces. A glass needle was inserted through a small nick in the neural tube of the embryo and a small portion of the neural tube was removed at brachial or thoracic levels. GFP ${ }^{+}$EBs were injected into this lesion site. Embryos were allowed to develop for 2-5 days post transplantation and then collected, fixed and prepared for cryosections or vibratome sections.

Retrograde labelling of transplanted MNs. Hb9::GFP and Hb9::Foxp1 ESCderived MNs were transplanted into the chick spinal cord at brachial levels. About 3 days later embryos with $\mathrm{GFP}^{+}$cells were identified and used for retrograde tracing experiments. Dextran (Biotinylated 3,000 MW; $10 \mathrm{mg} \mathrm{ml}^{-1}$; Invitrogen) was injected into the axial or limb nerve branches. Embryos were cultured at $30^{\circ} \mathrm{C}$ for $6-7 \mathrm{~h}$, fixed and prepared for cryosections.

Human ESC culture and differentiation to MNs. All human ESC experiments were conducted with prior approval from the UCLA Embryonic Stem Cell Research Oversight (ESCRO) Committee. H9 human ESCs (Madison, Wisconsin) were maintained on irradiated MEFs in human ESC media (DMEM/F12, 20\% knockout serum replacement, $1 \%$ nonessential amino acids, $1 \%$ glutamax, $0.1 \mathrm{mM}$ 2-mercaptoethanol and $10 \mathrm{ng} \mathrm{ml}^{-1}$ of basic fibroblast growth factor). Human ESCs were differentiated to MNs as previously described ${ }^{14}$. RA was added to the human ESC-derived neural progenitors after 10 days of differentiation. Neural rosettes were manually isolated and transferred into neural differentiation media with $0.1 \mu \mathrm{M}$ RA and $1 \mu \mathrm{M} \mathrm{SAG}$. After 28 days, human ESC-derived MN progenitors were cultured in human MN maturation media with $0.1 \mu \mathrm{M}$ RA, $50 \mathrm{nM}$ SAG, cyclic AMP $\left(0.1 \mu \mathrm{M}\right.$; Sigma), ascorbic acid (200 $\mathrm{ng} \mathrm{ml}^{-1}$; Sigma), insulin growth factor 1 (10 $\mathrm{n} \mathrm{ml}^{-1}$; Peprotech) and NFs (GDNF, BDNF and CNTF; all $10 \mathrm{ng} \mathrm{ml}^{-1}$; Peprotech)). For immunostaining, hESC-derived MNs were lightly dissociated using 20\% TrypLE (Invitrogen) and plated on matrigel-coated glass coverslips in MN maturation media. 
Lentiviral transduction of human ESC-derived MNs. The coding sequence of human FOXP1 (isoform 1) was PCR amplified from the plasmid template HsCD00297105 (PlasmID Database, Dana Farber/Harvard Cancer Center DNA Resource Core), and then cloned into the FUCRW lentiviral vector ${ }^{66}$. Control FUCRW (RFP) and FUCRW-FOXP1 lentiviruses were generated using standard third generation protocols, and concentrated viral aliquots were stored at $-80^{\circ} \mathrm{C}$. Newly born human ESC-derived MNs ( $~ 35$ days after start of differentiation) were infected overnight with the concentrated lentiviruses. $\mathrm{RFP}^{+}$cells were identified 3-5 days after infection. About 1-2 weeks after infection, transduced human ESC-derived MNs were fixed in 4\% paraformaldehyde for $10 \mathrm{~min}$ at room temperature before being processed for immunostaining.

Quantification. For analysis of MN populations in E12.5 mouse embryos: mean \pm s.e.m. values were calculated by pooling at least three sections at both cervical and thoracic levels, collected from at least three embryos of each genotype. For analysis of ESC-derived MNs: 3-5 field images containing $\sim 100$ cells per field were counted for each antibody stain per differentiation batch. Values from three separate differentiation batches were averaged to generate mean \pm s.e.m. values shown in the figures.

Statistics. No statistical methods were used to predetermine sample sizes, but our sample sizes are similar to those reported in previous publications ${ }^{13,19}$. All quantification of in vitro $\mathrm{MN}$-muscle co-cultures and explants was performed blind. Blinding of other experiments and randomization were not performed. Data were not tested for normality and compared using either the Student's $t$-test, a paired two-tail $t$-test, or one or two-way analysis of variance with Bonferroni adjustments for multiple comparisons. Variance was similar between groups being compared. The test used for each experiment is noted in the figure legends. All statistics and graphs were generated using Prism6 Graphpad software.

\section{References}

1. Faravelli, I. et al. Motor neuron derivation from human embryonic and induced pluripotent stem cells: experimental approaches and clinical perspectives. Stem Cell Res. Ther. 5, 87 (2014).

2. Frattini, E. et al. Pluripotent stem cell-based models of spinal muscular atrophy. Mol. Cell. Neurosci. 64C, 44-50 (2014).

3. Richard, J. P. \& Maragakis, N. J. Induced pluripotent stem cells from ALS patients for disease modelling. Brain Res. doi: 10.1016/j.brainres.2014.09.017 (2014).

4. Sandoe, J. \& Eggan, K. Opportunities and challenges of pluripotent stem cell neurodegenerative disease models. Nat. Neurosci. 16, 780-789 (2013).

5. Winner, B., Marchetto, M. C., Winkler, J. \& Gage, F. H. Human-induced pluripotent stem cells pave the road for a better understanding of motor neuron disease. Hum. Mol. Genet. 23, R27-R34 (2014).

6. Soundararajan, P., Miles, G. B., Rubin, L. L., Brownstone, R. M. \& Rafuse, V. F. Motoneurons derived from embryonic stem cells express transcription factors and develop phenotypes characteristic of medial motor column neurons. J. Neurosci. 26, 3256-3268 (2006).

7. Dalla Torre di Sanguinetto, S. A., Dasen, J. S. \& Arber, S. Transcriptional mechanisms controlling motor neuron diversity and connectivity. Curr. Opin. Neurobiol. 18, 36-43 (2008).

8. Kanning, K. C., Kaplan, A. \& Henderson, C. E. Motor neuron diversity in development and disease. Annu. Rev. Neurosci. 33, 409-440 (2010).

9. Butler, S. J. \& Bronner, M. E. From classical to current: Analyzing peripheral nervous system and spinal cord lineage and fate. Dev. Biol. 398, 135-146 (2014).

10. Francius, C. \& Clotman, F. Generating spinal motor neuron diversity: a long quest for neuronal identity. Cell. Mol. Life Sci. 71, 813-829 (2014).

11. Davis-Dusenbery, B. N., Williams, L. A., Klim, J. R. \& Eggan, K. How to make spinal motor neurons. Development 141, 491-501 (2014).

12. Dasen, J. S., De Camilli, A., Wang, B., Tucker, P. W. \& Jessell, T. M. Hox repertoires for motor neuron diversity and connectivity gated by a single accessory factor, FoxP1. Cell 134, 304-316 (2008).

13. Rousso, D. L., Gaber, Z. B., Wellik, D., Morrisey, E. E. \& Novitch, B. G. Coordinated actions of the forkhead protein Foxp1 and Hox proteins in the columnar organization of spinal motor neurons. Neuron 59, 226-240 (2008).

14. Hu, B. Y. \& Zhang, S. C. Differentiation of spinal motor neurons from pluripotent human stem cells. Nat. Protoc. 4, 1295-1304 (2009).

15. Wichterle, H., Lieberam, I., Porter, J. A. \& Jessell, T. M. Directed differentiation of embryonic stem cells into motor neurons. Cell 110, 385-397 (2002).

16. Wichterle, H. \& Peljto, M. Differentiation of mouse embryonic stem cells to spinal motor neurons. Curr. Protoc. Stem Cell Biol. Chapter 1, Unit 1H $11-1 \mathrm{H}$ $19 \mathrm{HH}(2008)$

17. Qu, Q. et al. High-efficiency motor neuron differentiation from human pluripotent stem cells and the function of Islet-1. Nat. Commun. 5, 3449 (2014).

18. Patani, R. et al. Retinoid-independent motor neurogenesis from human embryonic stem cells reveals a medial columnar ground state. Nat. Commun. 2, 214 (2011).
19. Peljto, M., Dasen, J. S., Mazzoni, E. O., Jessell, T. M. \& Wichterle, H. Functional diversity of ESC-derived motor neuron subtypes revealed through intraspinal transplantation. Cell Stem Cell 7, 355-366 (2010).

20. Surmeli, G., Akay, T., Ippolito, G. C., Tucker, P. W. \& Jessell, T. M. Patterns of spinal sensory-motor connectivity prescribed by a dorsoventral positional template. Cell 147, 653-665 (2011).

21. Rousso, D. L. et al. Foxp-mediated suppression of N-cadherin regulates neuroepithelial character and progenitor maintenance in the CNS. Neuron 74, 314-330 (2012).

22. Sockanathan, S. \& Jessell, T. M. Motor neuron-derived retinoid signaling specifies the subtype identity of spinal motor neurons. Cell 94, 503-514 (1998)

23. Vrieseling, E. \& Arber, S. Target-induced transcriptional control of dendritic patterning and connectivity in motor neurons by the ETS gene Pea3. Cell 127, 1439-1452 (2006).

24. Lacombe, J. et al. Genetic and functional modularity of Hox activities in the specification of limb-innervating motor neurons. PLoS Genet. 9, e1003184 (2013).

25. Dasen, J. S., Tice, B. C., Brenner-Morton, S. \& Jessell, T. M. A Hox regulatory network establishes motor neuron pool identity and target-muscle connectivity. Cell 123, 477-491 (2005)

26. Haase, G. et al. GDNF acts through PEA3 to regulate cell body positioning and muscle innervation of specific motor neuron pools. Neuron 35, 893-905 (2002).

27. Machado, C. B. et al. Reconstruction of phrenic neuron identity in embryonic stem cell-derived motor neurons. Development 141, 784-794 (2014).

28. Philippidou, P., Walsh, C. M., Aubin, J., Jeannotte, L. \& Dasen, J. S. Sustained Hox 5 gene activity is required for respiratory motor neuron development. Nat. Neurosci. 15, 1636-1644 (2012).

29. Dasen, J. S., Liu, J. P. \& Jessell, T. M. Motor neuron columnar fate imposed by sequential phases of Hox-c activity. Nature 425, 926-933 (2003).

30. Kania, A. \& Jessell, T. M. Topographic motor projections in the limb imposed by LIM homeodomain protein regulation of ephrin-A:EphA interactions. Neuron 38, 581-596 (2003).

31. Helmbacher, F., Schneider-Maunoury, S., Topilko, P., Tiret, L. \& Charnay, P. Targeting of the EphA4 tyrosine kinase receptor affects dorsal/ventral pathfinding of limb motor axons. Development 127, 3313-3324 (2000).

32. Kramer, E. R. et al. Cooperation between GDNF/Ret and ephrinA/EphA4 signals for motor-axon pathway selection in the limb. Neuron 50, 35-47 (2006).

33. Luria, V., Krawchuk, D., Jessell, T. M., Laufer, E. \& Kania, A. Specification of motor axon trajectory by ephrin-B:EphB signaling: symmetrical control of axonal patterning in the developing limb. Neuron 60, 1039-1053 (2008).

34. Ebens, A. et al. Hepatocyte growth factor/scatter factor is an axonal chemoattractant and a neurotrophic factor for spinal motor neurons. Neuron 17, 1157-1172 (1996).

35. Yamamoto, Y. et al. Hepatocyte growth factor $(\mathrm{HGF} / \mathrm{SF})$ is a muscle-derived survival factor for a subpopulation of embryonic motoneurons. Development 124, 2903-2913 (1997).

36. Shirasaki, R., Lewcock, J. W., Lettieri, K. \& Pfaff, S. L. FGF as a target-derived chemoattractant for developing motor axons genetically programmed by the LIM code. Neuron 50, 841-853 (2006).

37. Egea, J. \& Klein, R. Bidirectional Eph-ephrin signaling during axon guidance. Trends Cell Biol. 17, 230-238 (2007).

38. Sendtner, M., Pei, G., Beck, M., Schweizer, U. \& Wiese, S. Developmental motoneuron cell death and neurotrophic factors. Cell Tissue Res. 301, 71-84 (2000).

39. Hadas, Y. et al. A 'tool box' for deciphering neuronal circuits in the developing chick spinal cord. Nucleic Acids Res. 42, e148 (2014).

40. Arber, S. Motor circuits in action: specification, connectivity, and function. Neuron 74, 975-989 (2012).

41. Amoroso, M. W. et al. Accelerated high-yield generation of limb-innervating motor neurons from human stem cells. J. Neurosci. 33, 574-586 (2013).

42. Arber, S. et al. Requirement for the homeobox gene $\mathrm{Hb} 9$ in the consolidation of motor neuron identity. Neuron 23, 659-674 (1999).

43. Thaler, J. et al. Active suppression of interneuron programs within developing motor neurons revealed by analysis of homeodomain factor HB9. Neuron 23, 675-687 (1999).

44. Hester, M. E. et al. Rapid and efficient generation of functional motor neurons from human pluripotent stem cells using gene delivered transcription factor codes. Mol. Ther. 19, 1905-1912 (2011).

45. Son, E. Y. et al. Conversion of mouse and human fibroblasts into functional spinal motor neurons. Cell Stem Cell 9, 205-218 (2011).

46. Theys, P. A., Peeters, E. \& Robberecht, W. Evolution of motor and sensory deficits in amyotrophic lateral sclerosis estimated by neurophysiological techniques. J. Neurol. 246, 438-442 (1999).

47. O'Brien, M. K., Landmesser, L. \& Oppenheim, R. W. Development and survival of thoracic motoneurons and hindlimb musculature following transplantation of the thoracic neural tube to the lumbar region in the chick embryo: functional aspects. J. Neurobiol. 21, 341-355 (1990). 
48. O’Brien, M. K. \& Oppenheim, R. W. Development and survival of thoracic motoneurons and hindlimb musculature following transplantation of the thoracic neural tube to the lumbar region in the chick embryo: anatomical aspects. J. Neurobiol. 21, 313-340 (1990).

49. Wang, B. et al. Foxp1 regulates cardiac outflow tract, endocardial cushion morphogenesis and myocyte proliferation and maturation. Development 131, 4477-4487 (2004).

50. Nagy, A., Gertsenstein, M., Vintersten, K. \& Behringer, R. Collecting zygotes and removing cumulus cells with hyaluronidase. CSH Protoc. 2006 doi: 10.1101/pdb.prot4358 (2006).

51. Nagy, A., Gertsenstein, M., Vintersten, K. \& Behringer, R. De Novo isolation of embryonic stem (ES) cell lines from blastocysts. CSH Protoc. 2006 doi: 10.1101/ pdb.prot4403 (2006).

52. Umbach, J. A., Adams, K. L., Gundersen, C. B. \& Novitch, B. G. Functional neuromuscular junctions formed by embryonic stem cell-derived motor neurons. PLoS ONE 7, e36049 (2012).

53. Wichterle, H., Peljto, M. \& Nedelec, S. Xenotransplantation of embryonic stem cell-derived motor neurons into the developing chick spinal cord. Methods Mol. Biol. 482, 171-183 (2009).

54. Gaber, Z. B., Butler, S. J. \& Novitch, B. G. PLZF regulates fibroblast growth factor responsiveness and maintenance of neural progenitors. PLoS Biol. 11, e1001676 (2013).

55. Ji, S. J. et al. Mesodermal and neuronal retinoids regulate the induction and maintenance of limb innervating spinal motor neurons. Dev. Biol. 297, 249-261 (2006).

56. Arber, S., Ladle, D. R., Lin, J. H., Frank, E. \& Jessell, T. M. ETS gene Er81 controls the formation of functional connections between group Ia sensory afferents and motor neurons. Cell 101, 485-498 (2000).

57. Ericson, J., Briscoe, J., Rashbass, P., van Heyningen, V. \& Jessell, T. M. Graded sonic hedgehog signaling and the specification of cell fate in the ventral neural tube. Cold Spring Harb. Symp. Quant. Biol. 62, 451-466 (1997).

58. Tsuchida, T. et al. Topographic organization of embryonic motor neurons defined by expression of LIM homeobox genes. Cell 79, 957-970 (1994).

59. Bader, D., Masaki, T. \& Fischman, D. A. Immunochemical analysis of myosin heavy chain during avian myogenesis in vivo and in vitro. J. Cell Biol. 95, 763-770 (1982).

60. De Marco Garcia, N. V. \& Jessell, T. M. Early motor neuron pool identity and muscle nerve trajectory defined by postmitotic restrictions in Nkx6.1 activity. Neuron 57, 217-231 (2008).

61. Ilia, M. et al. Expression of Oct-6, a POU III domain transcription factor, in schizophrenia. Am. J. Psychiatry 159, 1174-1182 (2002).

62. Matthew, W. D., Tsavaler, L. \& Reichardt, L. F. Identification of a synaptic vesicle-specific membrane protein with a wide distribution in neuronal and neurosecretory tissue. J. Cell Biol. 91, 257-269 (1981).

63. Trevarrow, B., Marks, D. L. \& Kimmel, C. B. Organization of hindbrain segments in the zebrafish embryo. Neuron 4, 669-679 (1990).
64. Beurg, M. et al. Control of exocytosis by synaptotagmins and otoferlin in auditory hair cells. J. Neurosci. 30, 13281-13290 (2010).

65. Hazen, V. M., Phan, K., Yamauchi, K. \& Butler, S. J. Assaying the ability of diffusible signaling molecules to reorient embryonic spinal commissural axons. J. Vis. Exp. doi: 10.3791/1853 (2010).

66. Memarzadeh, S. et al. Enhanced paracrine FGF10 expression promotes formation of multifocal prostate adenocarcinoma and an increase in epithelial androgen receptor. Cancer Cell 12, 572-585 (2007).

\section{Acknowledgements}

We thank S. Butler, A. Kania and W. Lowry for helpful discussion and comments on the manuscript; M. Bonner and K. Plath for experimental instruction; N. Brecha, A. Goldstein, C. Gunderson, T. Jessell, S. Memarzadeh, S. Pfaff, H. Wichterle and O. Witte for reagents; A. Han for collecting RNA from Foxp1 ${ }^{+/-}$and Foxp1 $1^{-1}$ embryonic spinal MNs. This work was supported by the UCLA Broad Center for Regenerative Medicine and Stem Cell Research, The Rose Hills Foundation and grants to B.G.N. from the NINDS (NS072804), Muscular Dystrophy Association (92901) and California Institute for Regenerative Medicine (RB1-01367). K.L.A. was supported by the UCLA Cellular and Molecular Biology Training program (Ruth L. Kirschstein NIH GM00785), the UCLA-California Institute for Regenerative Medicine Training Grant and a UCLA Graduate Division Dissertation Year Fellowship.

\section{Authors contributions}

K.L.A. and B.G.N. conceived and designed the experiments and wrote the manuscript. K.L.A. performed and analysed most of the experiments. D.L.R. generated the Hb9::Foxp1 transgenic mice. J.A.U. performed the electrophysiology experiments.

\section{Additional information}

Supplementary Information accompanies this paper at http://www.nature.com/ naturecommunications

Competing financial interests: The authors declare no competing financial interests

Reprints and permission information is available online at http://npg.nature.com/ reprintsandpermissions/

How to cite this article: Adams, K. L. et al. Foxp1-mediated programming of limb-innervating motor neurons from mouse and human embryonic stem cells. Nat Commun. 6:6778 doi: 10.1038/ncomms7778 (2015).

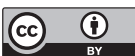

This work is licensed under a Creative Commons Attribution 4.0 International License. The images or other third party material in this article are included in the article's Creative Commons license, unless indicated otherwise in the credit line; if the material is not included under the Creative Commons license users will need to obtain permission from the license holder to reproduce the material. To view a copy of this license, visit http://creativecommons.org/licenses/by/4.0/ 